\title{
Faculty of Science
}

Faculty Publications

This is a post-print version of the following article:

Vertically Aligned Ni Nanowires as Platform for Kinetically-Limited Water Splitting Electrocatalysis

Minghui H. Hao, Sébastien Garbarino, Sagar Prabhudev, Tory Borsboom-Hanson, Gianluigi A. Botton, David A. Harrington and Daniel Guay

December 2018 (online)

This document is the Accepted Manuscript version of a Published Work that appeared in final form in The Journal of Physical Chemistry C, copyright (C) American Chemical Society after peer review and technical editing by the publisher. To access the final edited and published work see https://pubs.acs.org/articlesonrequest/AOR-jNwRNVxekMRdRNSJ8h2X

The final publication will be available at https://doi.org/10.1021/acs.jpcc.8b10414

Citation for this paper:

Hao, M.H., Garbarino, S., Prabhudev, S., Borsboom-Hanson, T., Botton, G.A., Harrington, D.A. \& Guay, D. (2018). Vertically Aligned Ni Nanowires as Platform for Kinetically-Limited Water Splitting Electrocatalysis. The Journal of Physical Chemistry C, 123(2), 1082-1093. https://doi.org/10.1021/acs.jpcc.8b10414 
This document is the Accepted Manuscript version of a Published Work that appeared in final form in J. Phys. Chem. C, 123 (2019) 1082-1093, copyright (C) American Chemical Society after peer review and technical editing by the publisher. To access the final edited and published work see https://pubs.acs.org/articlesonrequest/AOR-jNwRNVxekMRdRNSJ8h2X.

doi: $\underline{10.1021 / \text { acs.jpcc. } 8 \text { b10414 }}$

\section{Vertically Aligned Ni Nanowires as Platform for Kinetically-Limited Water Splitting Electrocatalysis}

\section{REVISED jp-2018-104143}

Minghui H. Hao ${ }^{1}$, Sébastien Garbarino ${ }^{1}$, Sagar Prabhudev², Tory Borsboom-Hanson ${ }^{3}$, Gianluigi A. Botton ${ }^{2}$, David A. Harrington ${ }^{3}$ and Daniel Guay ${ }^{1 *}$

${ }^{1}$ Institut national de la recherche scientifique Énergie, matériaux et télécommunications (INRS - EMT) 1650 Lionel Boulet Blvd. Varennes, QC, Canada J3X 1S2

${ }^{2}$ Department of Materials Science and Engineering and Canadian Centre for Electron Microscopy, McMaster University, 1280 Main Street West, Hamilton, Ontario, Canada L8S 4M4

${ }^{3}$ Department of Chemistry, University of Victoria, PO Box 1700, Victoria, BC, Canada V8W 2Y2

* Corresponding author Email: guay@emt.inrs.ca 


\begin{abstract}
Electrochemical Templating through porous membranes is applied to form arrays of micrometer long $\mathrm{Ni}$ nanowires $(\mathrm{NW})$. Detailed structural and electrochemical characterization, including Electrochemical Impedance Spectroscopy (EIS), were conducted to assess the electrocatalytic properties of these Ni NW arrays for the $\mathrm{O}_{2}$ evolution reaction (OER) in $1 \mathrm{M} \mathrm{KOH}$. Detailed structural analysis showed that Ni NWs have a diameter of $c a$ $350 \mathrm{~nm}$ and a mean $80 \mathrm{~nm}$ average distance between NWs center. For the longest NWs (20 $\mu \mathrm{m}$ long), the ratio between the pore opening and pore length is $0.4 \%$. From detailed HRTEM and EELS analysis providing information on the chemical state of atoms from quantitative analysis of the signals, Ni NWs are composed of a Ni metallic core surrounded by a $\mathrm{Ni}(\mathrm{OH})_{2}$ layer that thickens from $10 \mathrm{~nm}$ to $20 \mathrm{~nm}$ after extensive electrochemical test. Three different methods, namely SEM geometry measurements, the alpha-Ni(OH) 2 charge method and the capacitance method, were used to assess how the current varies with the NW length. The three different methods are all in agreement and the current increases with length (or mass) precisely and only because of the surface area effect. The most surprising result is that the OER process occurs with exactly the same intrinsic catalytic activity at the bottom of these deep pores and gas is evolved without any significant effects of electrolyte resistance, mass transport of dissolved oxygen, or bubble occlusion of the pores. Accordingly, vertically aligned 1D NWs can be used as an effective platform that mitigates the negative effects of gas evolution. Increasing the intrinsic activity by incorporating more active materials will further improve this type of electrode.
\end{abstract}




\section{Introduction}

The promise of hydrogen as a sustainable, secure, and clean alternative energy carrier to satisfy growing global energy demand requires efficient, low-cost, and environmentally friendly production. Water electrolysis to generate hydrogen fuel - preferably driven by renewable energy is a highly attractive means to produce hydrogen but advanced electrolyzer systems are required for electrolytic $\mathrm{H}_{2}$ production to become a commercially viable technologies. Lately, new classes of anion exchange membranes (AEM) have emerged with improved performance over their predecessors, ${ }^{1-5}$ improving the future prospect of commercialized Anionic Polymer Electrolyte Membrane Water Electrolysers (APEMWEs).

The prospect of new AEMs for alkaline APEMWEs that could match the performances of cationic exchange membranes in terms of ionic conductivity and longevity has spurred a renewed interest in research to improve catalysts performance for the Oxygen Evolution Reaction (OER) in alkaline electrolyte. Recent papers in the literature have emphasized the critical importance of electrode composition in achieving high OER performance. Indeed, it turns out that pure Ni (with a $\mathrm{Ni}(\mathrm{OH})_{2}$ film) is a rather poor catalysts for the OER. This was known for a long time since the seminal work of Corrigan ${ }^{6}$ but it was re-emphasized only recently ${ }^{7}$. Indeed, in rigorously Fe-free $\mathrm{KOH}$ electrolyte, Ni electrodes exhibit large overpotentials for the OER that is considerably reduced by trace amount of Fe. The amount of Fe needed to "dope" Ni depends on the electrochemically active surface area of the electrode, ${ }^{6}$ and this has prompted a plethora of work aiming at finding the composition of Ni-based electrodes that would give optimal OER performances in terms of overpotential, current density and stability. This work has recently been reviewed..$^{8-10}$

Another thrust of research is focusing on imparting the electrode surface with nanoengineering characteristics to improve OER performance by decreasing the bubbles' size, residence 
time, and detachment force through electrode surface nano-engineering. The adhesion force between a bubble and a solid originates from the triple phase (solid-liquid-gas) contact line. On a flat surface, this triple phase contact line is continuous, while it becomes discontinuous and thus less important on a nanostructured surface. This translates into smaller bubble size and lower bubble adhesion force, thereby leading to improved electrochemical performances. These surfaces are known as "super-aerophobic" and several materials and electrodes have been investigated showing improved performance for electrolytic gas evolution reactions like the OER and the hydrogen evolution reaction (HER) upon nanostructuring of the electrode surface, namely Ni, ${ }^{11} \mathrm{MoS}_{2},{ }^{12}$ NiMo and NiFe-layered double hydroxide, ${ }^{13} \mathrm{Ni} / \mathrm{Co}$ oxide, $,{ }^{14} \mathrm{NiCoP},{ }^{15} \mathrm{CoS}^{16}$ and $\mathrm{Pt} .{ }^{17}$

Nanostructuring of the electrode surface through the creation of pores and cavities that extend to the bulk of the electrode (3D nanostructured electrode) increases the number of active sites accessible to the electrolyte, thereby increasing the current density at fixed overpotential. However, apart from good electronic conductivity, the electrode 3D structure must also not impose any masstransport limitation of dissolved oxygen or ionic migration within the pores. Ideally, the OER of the optimal 3D nanostructured electrode must be kinetically-controlled in the same overpotential range as that observed on Ni thin films.

In this study, we synthesized arrays of micrometer long Ni nanowires with high surface area to ensure that OER current densities could be reached at low overpotentials. To do so, we used a template-assisted Ni electrodeposition procedure to prepare electrodes made of 1D Ni nanowires densely packed in a brush-like array structure. Careful structural characterization of pristine electrode indicates that $\mathrm{Ni}$ nanowires are covered with a thin $\mathrm{Ni}(\mathrm{OH})_{2}$ layer $(c a .10 \mathrm{~nm})$ that thickens by a factor of $2(c a .20 \mathrm{~nm})$ after extensive use for the OER. Cyclic voltammetry, chronopotentiometry and Electrochemical Impedance Spectroscopy (EIS) measurements under $\mathrm{O}_{2}$ 
evolving conditions indicate that both the electrochemically active surface area (measured by cyclic voltammetry in quiescent conditions) and the effective capacitance (measured in a potential region where the OER is occurring) scales with the nanowire length and calculated surface area, indicating full accessibility. This translates into the OER being kinetically-limited, instead of diffusion-limited, even if the pore aspect ratio, which is defined as the ratio between the pore opening and pore length, is $0.4 \%$. The overpotential at $10 \mathrm{~mA} \mathrm{~cm}^{-2}$ for the longest Ni NWs $(20 \mu \mathrm{m})$ is $\approx 200 \mathrm{mV}$ lower than on a bare Ni plate.

\section{Experimental}

\subsection{Synthesis of porous Ni electrodeposits}

Galvanostatic Ni electrodeposition $\left(10 \mathrm{~mA} \mathrm{~cm}{ }^{-2}\right)$ was performed from aqueous solutions of $0.1 \mathrm{M} \mathrm{NiCl}_{2} \cdot 6 \mathrm{H}_{2} \mathrm{O}$ (Alfa Aesar, 99.95\% Metals Basis) and $2 \mathrm{M} \mathrm{NH}_{4} \mathrm{Cl}$ (Sigma-Aldrich, 99.99\% Trace Metals Basis) through a porous Aluminum Anodic Oxide (AAO) membrane (Anodisc25, Whatman International Ltd.). A titanium layer $(400 \mathrm{~nm})$ was DC sputtered at the AAO backside to ensure electrical contact mandatory for Ni electroplating. A saturated calomel electrode (SCE) and Pt gauze (Alfa Aesar, 99.9\%) were used as reference and counter electrodes, respectively. Ni electrodeposition was carried out using a Solartron 1480 for durations up to 3 hours. Following Ni electroplating, the AAO membrane was dissolved in $1 \mathrm{M} \mathrm{KOH}$ for $1 \mathrm{~h}$ at room temperature, thoroughly rinsed with de-ionized water (Millipore Milli-Q, resistivity $18.2 \mathrm{M} \Omega \mathrm{cm}$ ) and immediately characterized electrochemically. All the potentials measured in $1 \mathrm{M} \mathrm{KOH}$ are reported vs RHE using $E_{\mathrm{RHE}}=E_{\mathrm{SCE}}+1.04 \mathrm{~V}$. The geometric surface of the Ni working electrodes was 0.15 $\mathrm{cm}^{2}$ in all cases.

\subsection{Physical characterization of porous Ni electrodeposits}


TEM observations were carried out with a FEI Titan 80-300 Cubed Scanning Transmission Electron Microscope equipped with a high-brightness field-emission electron source and ultra-fast (electron energy loss) EEL Spectrometer. In cases where a cross-sectional analyses of the NW structure needed to be carried out, an ultra-thin area was carefully lift out using Focused Ion Beam (FIB) technique (see Supporting Figure S1). High resolution SEM analyses were carried out using the FEI-Magellan SEM microscope. Detailed elemental mapping necessary to resolve the spatial distribution of Ni and $\mathrm{O}$ was carried out using the Electron Energy Loss Spectroscopy (EELS) Spectrum Imaging (SI) technique in the TEM. The process involved a serial acquisition of the EEL Spectra at every pixel on the annular dark field (ADF) image and subsequent processing to extract the Ni-L2,3, O-K and Ti-L2,3 edges. Mapping the spatial distribution of these individual edges revealed the relative distribution of $\mathrm{Ni}, \mathrm{O}$ and $\mathrm{Ti}$ elements, respectively. To further determine the chemical state of $\mathrm{Ni}$ in certain NW samples the comparison of their $\mathrm{Ni}-\mathrm{L}_{3} / \mathrm{L}_{2}$ white-line intensity ratios was carried out, involving the subtraction of the continuum background under the power-law background stripped EELS spectrum by fitting a Double ArcTan function. This procedure is considered accurate at estimating $\mathrm{L}_{3} / \mathrm{L}_{2}$ ratios of white lines. ${ }^{18}$

\subsection{Electrochemical characterization and OER performances}

Electrochemical characterization in Ar-saturated (N50, Air Liquide) 1 M KOH (SigmaAldrich, 99.99\% Trace Metals Basis) was conducted in a custom-built electrochemical cell, ${ }^{19}$ using a Pt gauze and a saturated calomel electrode as auxiliary and reference electrode, respectively. Cyclic voltammograms (-0.10 V to $0.45 \mathrm{~V}$ vs RHE, $\left.25 \mathrm{mV} \mathrm{s}^{-1}\right)$ were first recorded until steady-state CV features were obtained. Then the electrodes were subjected to 10 minutes open circuit potential (OCP) before chronopotentiometry curves were recorded (10 $\mathrm{mA} \mathrm{cm}{ }^{-2}$ for $\left.30 \mathrm{~min}\right)$. The above sequence was systematically applied to all of the reported Ni NW electrodes. The results were 
compared with a commercial Ni plate (Sigma Aldrich, thickness $0.125 \mathrm{~mm}, \geq 99.9 \%$ metals basis) and with electrodeposited Ni films obtained without any AAO membrane on Ti foil (Alfa Aesar, 99\% metals basis). The solution resistance was measured by Electrochemical Impedance Spectroscopy (EIS) and used to correct potentials for ohmic drops. EIS measurements in the OER potential range were obtained following 10 min polarization (from 1.5 to $1.6 \mathrm{~V}$ ) between $0.1 \mathrm{~Hz}$ and $200 \mathrm{kHz}$ using a FRA Analyser (Solartron 1255B). Electrochemical impedance spectra were fitted to equivalent circuit models using the complex nonlinear least squares (CNLS) fitting program ZVIEW (Scribner Associates).

\section{RESULTS}

\subsection{Growth of Ni nanowires and morphology}

Figure 1 illustrates the top-view and cross-sectional views of the typical pristine Ni nanowires (Ni NWs) synthesized. The low-magnification SEM image (Figure 1a) reveals a relatively dense packing of the NW bundles resembling that of a brush-like array structure. Detailed high-resolution SEM image (as shown in Figure 1b) indicates that some NWs have an oblong crosssection (small diameter $=310 \mathrm{~nm}$ and large diameter $=410 \mathrm{~nm}$ ), but most of them have a circular cross-section. Figure 1c illustrates the NW structure in cross-section. A size measurement along the length of the NW indicates that the pristine NWs synthesized were typically about 12 micrometers long.

Above Ni NWs were prepared by deposition using a porous AAO template membrane. Figure 2a displays SEM micrograph of the as-received AAO membrane, which shows the expected close to hexagonal assembly of pore arrangement. The size of the openings was assessed by measuring the diameter of a collection of more than 100 pores (see Figure 2a-inset). The mean pore 
size is $184 \pm 30 \mathrm{~nm}$. Then, Ni was deposited using the setup described in the experimental section, and Figure $2 \mathrm{~b}$ shows the SEM micrograph of the resulting Ni filled membrane (before the AAO membrane was dissolved). The deposition time was adjusted to limit the deposition charge and to prevent $\mathrm{Ni}$ deposition on top of the AAO membrane. The hexagonal pore arrangement is preserved. The mean pore size is $197 \pm 21 \mathrm{~nm}$ (see Figure $2 \mathrm{~b}$-inset). Under our deposition conditions, slow dissolution of the AAO template in acidic medium, which could have resulted in an increase of the pore size and/or modification of the hexagonal arrangement of pores, is negligible.

Then, the AAO membrane was dissolved in $1 \mathrm{M} \mathrm{KOH}$ and Figure 2c depicts the Ni structures that have grown inside the AAO pores. As seen in Figure 2c, the Ni NWs are arranged as nanostructured filaments that mimic the pore structure of the as-received AAO membrane. Finally, the pristine Ni NWs synthesized were subjected to an extensive electrochemical characterization in alkaline media. As seen in the corresponding SEM image shown in Figure 2d, the hexagonal structure of Ni NWs is again preserved and is not affected even if extensive polarization in the potential region where $\mathrm{O}_{2}$ evolution has occurred. The mean diameter of Ni NWs in this case is estimated to be about $354 \pm 38 \mathrm{~nm}$ (Figure $2 \mathrm{~d}$ - inset), which is fairly close to that of the pristine samples (i.e., NWs in Figure 1, $305 \pm 38 \mathrm{~nm}$ ).

Figure 3 compares the high-resolution SEM images of these last two samples. As seen, the morphological features remained remarkably stable and did not significantly evolve following extensive electrochemical characterization. An estimation of packing fraction in Ni-NW array electrodes with segmentation analyses of the SEM images was performed (Figure S3). Both square and hexagonal packing of NWs were identified in pristine and electrochemically treated Ni NWs. The estimated packing fractions of pristine ( $82 \%)$ and electrochemically treated $(84 \%)$ Ni NWs are almost identical and fall in between the values expected for ideally arranged square- (79\%) and 
hexagonal- (91\%) packing arrangement. The average spacing between two nanowires is $c a .80 \mathrm{~nm}$ in both cases. For the longest Ni NWs investigated in the present study (ca. $20 \mu \mathrm{m})$, the ratio between the average NW-NW spacing and NW length, which defined the aspect ratio of the openings in the Ni NW array electrode, varies from $2.7 \%$ for the smallest to $0.4 \%$ for the longest NWs.

Cross-section and top view SEM micrographs of Ni NW arrays prepared at different deposition times are shown in Figure S3. In each case, the samples are made of densely packed NWs arranged in a brush-like array. Ni NWs have a uniform diameter from bottom to top, which is expected since the diameter of the AAO pores does not vary between Figure 2a and 2b. As far as we can tell from these micrographs, there are very few missing NWs. The NW length increases steadily as the deposition time is increased from 10 to 180 minutes. Indeed, Figure S3E shows that the NW length increases linearly with deposition time. Current efficiency values were computed for each sample. This was done by taking the ratio between the charge involved in Ni deposition and the total charge. The former was calculated by assuming that the mass of Ni contained in an assembly of Ni NWs can be obtained from the known pore diameter, length and NW surface density, using Ni bulk density and a $2 \mathrm{e}$ - process. The deposition current efficiency is $c a .13 \%$, and independent of NW length (electrodeposition time).

Figure 4(a) illustrates the cross-sectional view of the pristine Ni NWs discussed earlier in Figure 1. As evidenced by the EELS elemental mapping of the Ni-L2,3, O-K and Ti- $\mathrm{L}_{2,3}$ edges in Figure 4(b), the NWs seem to nucleate at the contact of the $\mathrm{TiO}_{\mathrm{x}}$ substrate and grow upward along the initial AAO template. An exception to this rule are regions such as that highlighted in yellow in Figure 4(b) where the metallic-Ni (depleted of both $\mathrm{O}$ and $\mathrm{Ti}$ ) can be seen extending all along the $\mathrm{TiO}_{\mathrm{x}}$ substrate down to the bottom. This suggests that the nucleation of $\mathrm{Ni}$ and the further growth of the NW can possibly also take place in the absence of a $\mathrm{TiO}_{x}$ phase. Unravelling this hypothesis 
would, however, require further modelling of the energetic considerations of the growth process that is beyond the scope of the present article.

Similar to the above analyses, we also carried out a detailed compositional analysis of the top and the middle regions of the NWs. As seen in the Figure 4(c, d), both the top- and the middleregions show significant enrichment in the $\mathrm{O}$ species at the surface (see Table 1). This suggests that the NW surfaces formed an oxide layer over the metallic core and knowing the exact phase would be important from an electrochemical stability viewpoint (see further below for a detailed discussion on this). It is noteworthy to mention here that the oxide layer was still preserved even after exposing the NWs to extensive electrochemical characterization $(29.4$ at. $\%$ vs. 26.7 at.\% in Oxygen concentration was detected at the NW surfaces before and after, respectively). Also to be noted here is that a close observation of the Ti maps in Figure $4 \mathrm{~b}$ (NW bottom) and $4 \mathrm{c}$ (NW middle region) reveals trace Ti- $\mathrm{L}_{2,3}$ signals in the near-surface regions, suggesting some possible inclusions of the Ti structure mostly on the surface of the NW structure (see Ti signal map in figure 4c). A thorough quantification of the $\mathrm{Ti} / \mathrm{O} / \mathrm{Ni}$ in these regions confirmed that such inclusions of $\mathrm{Ti}$ into the lattice is negligibly small ( $<1$ at.\%) particularly in the middle region of the NWs, but with an expected small level $\left(<10\right.$ at.\%) of inclusions in the regions closer to the $\mathrm{TiO}_{\mathrm{x}}$ substrate (see Table 1 for the quantified elemental concentrations).

In order to deduce the thermodynamic phase of the oxide layer formed (i.e., $\mathrm{NiO}$ vs. $\left.\mathrm{Ni}(\mathrm{OH})_{2}\right)$ in the two samples (NWs pre-/post- treatment in $1 \mathrm{M} \mathrm{KOH}$ ), we estimated their Ni- $\mathrm{L}_{3} / \mathrm{L}_{2}$ white-line intensity ratios over the core- and surface- regions. These were then compared against the corresponding white-line ratios of $\mathrm{NiO}$ and $\mathrm{Ni}(\mathrm{OH})_{2}$ phases extracted from a detailed EELS analyses conducted separately. 
Figure 5(a(i), b(i)) illustrate the core-/surface- regions within the two NW samples. Figures (ii, iii) shows the corresponding $\mathrm{Ni}$ edges from these regions. Note that the $\mathrm{Ni}$ edges shown have been stripped off of their original background using a power-law. The yellow continuum highlighted corresponds to the background not accounted for in the $\mathrm{L}_{3} / \mathrm{L}_{2}$ ratio estimation. The estimated $\mathrm{L}_{3} / \mathrm{L}_{2}$ white-line intensity ratios for the two NW samples along with the reference values for $\mathrm{NiO}, \mathrm{Ni}(\mathrm{OH})_{2}$ and metallic-Ni phases are tabulated in Table 2. As highlighted, the $\mathrm{L}_{3} / \mathrm{L}_{2}$ ratios in the core-region for both NW samples are almost identical - comparable to the metallic Ni state. Similarly, the $\mathrm{L}_{3} / \mathrm{L}_{2}$ ratios in the shell-region for both NW samples are similar - comparable to the $\mathrm{Ni}(\mathrm{OH})_{2}$ state. Accordingly, there is no obvious change in the chemical state of Ni following the extensive electrochemical characterization apart from a factor of 2 thickening of the $\mathrm{Ni}(\mathrm{OH})_{2}$ layer enclosing the metallic Ni core of each NW (see Figure S4). This is consistent with the fact that no obvious structural changes were detected from the detailed comparison of their high-resolution SEM images apart from a slight increase of the mean diameter from $305 \pm 36 \mathrm{~nm}$ to $354 \pm 38 \mathrm{~nm}$ (see Figure 3).

\subsection{Electrochemical characterization}

Electrochemically Active Surface Area (EASA), for the as-synthesised arrays of Ni NWs, was assessed by CVs in $1 \mathrm{M} \mathrm{KOH}$ at $25 \mathrm{mV} \mathrm{s}^{-1}$ (Figure 6A). The charge during the forward scan, $Q_{a}$, was used to evaluate the Ni EASA. Indeed, in this potential range (-0.10V and $\left.0.45 \mathrm{~V}\right)$, Ni can be reversibly oxidized to $\alpha-\mathrm{Ni}(\mathrm{OH})_{2}$ during the forward potential scan and reduced back to metallic $\mathrm{Ni}$ in the backward scan. ${ }^{20}$ Following 150 potential cycles, steady-state CV responses are obtained, with a representative example displayed in Figure 6. Measured $Q_{a}$ values were found to be proportional to the Ni NW length (Figure 7), which means that the reversible conversion Ni/ $\alpha$ $\mathrm{Ni}(\mathrm{OH})_{2}$ is occurring over the entire Ni NW length and that the overall Ni NW surface is accessible 


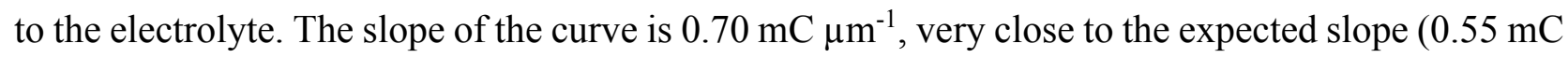
$\mu \mathrm{m}^{-1}$ ) based on pure geometrical considerations (ideal NW cylinders with similar diameter, length and surface density) and an assumption that the $\alpha-\mathrm{Ni}(\mathrm{OH})_{2}$ charge on an ideal surface is $0.514 \mathrm{mC}$ $\mathrm{cm}^{-2} \cdot{ }^{20}$ The difference between the predicted and measured values may arise from uncertainty in the calibration value or the measured diameters, the fact that the NW diameter varies slightly from bottom to top, and surface roughness at the nanoscale level (Figure 4). It is worth mentioning that the $\mathrm{Y}$-axis intercept of the straight line is different from zero. One reason for this could be that the bottom of each Ni NWs at the interface with the Ti thin film does not have a perfectly cylindrical shape.

Considerable changes occur in the $\mathrm{CV}$ of Ni NWs upon extension of the upper vertex potential limit to higher values. In the first CV following the restricted potential range used in Figure 6A, two anodic peaks at $0.23 \mathrm{~V}$ and $1.44 \mathrm{~V}$ were observed in the forward sweep and only one cathodic contribution at $1.34 \mathrm{~V}$ in the backward sweep. The upper quasi-reversible transition involves the well-known $\beta-\mathrm{Ni}(\mathrm{OH})_{2} / \beta-\mathrm{Ni}(\mathrm{OOH})$ nickel oxide charge storage process. The $10^{\text {th }}$ voltammetric profile no longer displays the anodic shoulder at $0.23 \mathrm{~V}$ (see Figure 6A and 6B), and both the anodic peak at $1.44 \mathrm{~V}$ and cathodic peak at $1.34 \mathrm{~V}$ are better defined than during the first $\mathrm{CV}$. These observations demonstrate that most of the $\alpha-\mathrm{Ni}(\mathrm{OH})_{2}$ species are no longer present, but have been converted to $\beta \mathrm{Ni}(\mathrm{OH})_{2}$ phases, which also results in a lower rate for the hydrogen evolution reaction. This ensures the measured $Q_{\mathrm{a}}$ coulombic charge was exclusively associated with the formation of $\alpha-\mathrm{Ni}(\mathrm{OH})_{2}$ species. Previous attempts to instead determine Ni EASA using the higher-potential $\mathrm{Ni}(\mathrm{II}) / \mathrm{Ni}(\mathrm{III})$ redox transition have been proposed, but uncertainty in the porosity and structure of these multilayer phases make the present procedure more reliable. 
The formation of Ni NWs translate into a huge increase of the EASA. Using $Q_{a}$ as measure, the EASA of a Ni plate and an electrodeposited Ni thin the same size $\left(0.15 \mathrm{~cm}^{2}\right)$ are 0.14 and 0.78 $\mathrm{mC}$, respectively, compared to $18.0 \mathrm{mC}$ for the $\mathrm{Ni} \mathrm{NWs}$ array sample with the longer $(19 \mu \mathrm{m})$ nanowires. This a factor of 130 and 10 increase of the EASA, respectively. In the following, we will focus our attention on assessing if the entire surface area of the nanowires is accessible to the electrolyte and able to participate in the oxygen evolution reaction or if mass-transport limitation of dissolved oxygen, ionic migration within the pores, or bubble occlusion effects are occurring, thereby limiting the benefits expected by increasing the EASA.

\subsection{Oxygen Evolution Reaction}

Chronopotentiometry profiles, with an applied current density of $10 \mathrm{~mA} \mathrm{~cm}{ }^{2}$ geo, were recorded in $1 \mathrm{M} \mathrm{KOH}$ for $30 \mathrm{~min}$ (Figure 8A). OER potentials at the Ni NW arrays continuously decrease with increasing Ni NW mean length, and initial potential values of $1.65 \mathrm{~V}$ and $1.60 \mathrm{~V}$ are obtained for $2.5 \mu \mathrm{m}$ and $19 \mu \mathrm{m}$ long Ni NWs, respectively. It is worth mentioning that any of the OER potential values observed at Ni NWs are lower than at electrodeposited Ni film (1.68 V), which confirms the enhanced initial OER activity at Ni NW electrodes. As time progresses the corresponding potential on any $\mathrm{Ni}$ electrode increases, especially for the $\mathrm{Ni}$ film obtained through electrodeposition without an AAO membrane, for which the potential rises to $1.83 \mathrm{~V}$. To obtain quantitative data on the OER stability, initial $(1 \mathrm{~min})$ and final potentials $(30 \mathrm{~min})$ are plotted in Figure 8B. It is straightforward to conclude that the longer $\mathrm{Ni} \mathrm{NW}$ electrodes are more stable for $\mathrm{O}_{2}$ evolution at $10 \mathrm{~mA} \mathrm{~cm}^{-2}$. The final potential values after $30 \mathrm{~min}$ electrolysis, are plotted in Figure 8C as function of $Q_{\mathrm{a}}$. A significant decrease of the OER potential was found for Ni NW with high

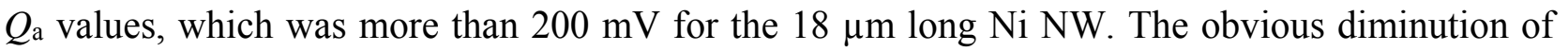
OER potential on longer Ni NW electrodes can be explained by the intrinsic current density which 
is lower for the larger EASA electrodes. Even though the OER potential may be affected by many other effects, the governing factor in this work is the enhanced Ni surface area for the ordered 1D nanostructures.

A Tafel analysis of Ni film, $3 \mu \mathrm{m}$-long and $17 \mu \mathrm{m}$-long Ni NW array electrodes at electrode potentials in the OER region is given in Figure 9. These points are the steady-state currents for potential steps, which are plotted with the $I R_{\mathrm{s}}$-corrected potentials. The Tafel slopes are $c a .58 \pm 10$ $\mathrm{mV} \mathrm{dec} \mathrm{d}^{-1}$ for the thin-film and NW electrodes, indicating a transfer coefficient of 1 . Literature values on flat $\mathrm{Ni}$ electrodes are $c a .46 \mathrm{mV} \mathrm{dec}{ }^{-1},{ }^{21-23}$ which is consistent with the present result considering the stated error and some uncertainty in the roughness factor. On hierarchical $\mathrm{Ni}(\mathrm{OH})_{2} \mathrm{NW}$ arrays with much smaller features than here, the reported Tafel slope was $123 \mathrm{mV} \mathrm{dec}{ }^{-1} \cdot{ }^{24}$ This result may not be directly comparable, since porosity effects may be increasing the Tafel slope. ${ }^{25}$ The agreement between the thin film and NW electrodes and the independence of the Tafel slopes with the Ni NW lengths, confirms that the OER is kinetically-controlled in this overpotential range, and that mass-transport of dissolved oxygen, ionic migration within the pores, or bubble occlusion effects are not significant.

\subsection{Electrochemical impedance spectroscopy on Ni NW}

Figure 10 shows the electrochemical impedance spectra of the electrodes at electrode potentials in the OER region, obtained after the steady-state measurements of Figure 9 were made. The EIS spectra of the Ni thin film electrode shows only one depressed semicircle. In comparison, the EIS data of Ni NWs display an additional semicircle at high frequencies. On Ni NWs, the shape of the high-frequency second semicircle does not change with the electrode potential, although its shape differs between short $(3 \mu \mathrm{m})$ and long $(17 \mu \mathrm{m}) \mathrm{Ni}$ NWs. This is an indication that the highfrequency semicircle is related to porosity and not to the electrode OER kinetics. The transmission- 
line model for porous electrodes comprising an array of identical pores has been well described. ${ }^{25-}$ ${ }^{27}$ In general, the theory predicts a smaller high-frequency, potential-independent feature related to the pore geometry, and a larger potential-dependent distorted semicircle related to the interfacial kinetics. C. Hitz and A. Lasia ${ }^{28}$ found experimentally and theoretically that for the hydrogen evolution reaction on porous $\mathrm{Ni}$, the porous electrode impedance may be closely approximated by two distorted semicircles modeled by two parallel resistor-CPE combinations in series. With the solution resistance $R_{\mathrm{S}}$ in series, this so-called 2CPE equivalent circuit was also found to fit the present results, which reflects the similar nature of the two systems: gas-evolving reactions at $\mathrm{Ni}$ porous electrodes in alkaline solution for which reactant $\left(\mathrm{H}_{2} \mathrm{O}\right.$ or $\left.\mathrm{OH}^{-}\right)$mass transport is fast. The dependence of the parameters of this model with the experimental conditions will be discussed, and then their relationship to the underlying porous electrode model.

In the case of the Ni thin film electrode, the circuit with only one parallel resistor-CPE combination "1CPE" was used (Figure 10A). The fits to the 2-CPE equivalent circuit for the OER at Ni NW electrodes are shown in Figures 10B and 10C. Before accepting this as the final equivalent circuit, we used the $F$-test ${ }^{29}$ to decide whether replacement of capacitors with CPEs was statistically justified. Acceptance required satisfying the $F$-test at the $99 \%$ confidence level and also that the error in the new fitted parameters was less than $30 \%$. Both CPEs were justified using these criteria. Similarly, addition of a Warburg element representing diffusion in the finite-length pores ("short Warburg") in series with $R_{\mathrm{ct}}$ was found not to be justified.

There is also the possibility that there are additional features, perhaps associated with slow mass transport of dissolved oxygen, which appear at frequencies lower than measured here. Lowerfrequency features associated with diffusion of solution species have been seen before for NW arrays, e.g., ${ }^{30,31}$ This is a particular concern for Nyquist plots in which only part of the low-frequency 
semicircle is observed, though inspection of complex capacitance plots that visually enhance lowfrequency features showed no low-frequency fine structure. The absence of lower frequency features can be verified by extrapolating the fitted impedance function to zero frequency, $Z(\omega \rightarrow 0)=R_{\mathrm{p}}$, and showing that it is equal to the slope of the steady-state polarization curve, $\mathrm{d} E / \mathrm{d} \mathrm{jss}_{\mathrm{ss}}{ }^{32}$ In the case that the Tafel plot is linear, this relationship may be transformed into

$$
\frac{R T}{\alpha F}=\frac{\mathrm{d} E}{\mathrm{~d}\left(\ln j_{\mathrm{ss}}\right)}=\frac{j_{\mathrm{ss}} \mathrm{d} E}{\mathrm{~d} j_{\mathrm{ss}}}=j_{\mathrm{ss}} R_{\mathrm{p}}
$$

i.e., the product of the steady state current and the low frequency impedance should be constant and when multiplied by $\ln (10)=2.303$ should equal the Tafel slope. For the $2 \mathrm{CPE}$ circuit, $\left(R_{1}+R_{\mathrm{ct}}\right) j_{\mathrm{ss}} \ln (10)$ was nearly constant $(60-90 \mathrm{mV})$, and very close to the $\left(I R_{\mathrm{s}}\right.$-corrected $)$ Tafel slope (see Figure S5), even though the current densities and $R_{\mathrm{ct}}$ each changed by more than an order of magnitude. In summary, the low-frequency relaxation is not significantly different from a parallel CPE resistor combination that represents the reaction kinetics, and there is no evidence for any significant mass-transport effects.

The fitted parameters are given in Table S1 and the fits show excellent accordance with the experimental results (Figure 10). Also given there are the effective capacitances ( $\left.C_{\text {eff }}\right)$ associated with a CPE, which were found using the Brug formula: ${ }^{33}$

$$
C_{\text {eff }}=\left[T_{2}\left(\frac{1}{R_{\mathrm{s}}}+\frac{1}{R_{\mathrm{ct}}}\right)^{\phi-1}\right]^{1 / \phi}
$$

An alternative form with $R_{S}$ replaced by $R_{S}+R_{1}$ was also evaluated but this made very little difference to the $C_{\text {eff values obtained. }}$

As the applied OER potential is increased, $R_{\text {ct }}$ continuously decreased for both $\mathrm{Ni}$ electrodeposited film and Ni NW electrodes, with an exponential (Tafel) potential dependence as 
expected since $R_{\mathrm{ct}} \approx R_{\mathrm{p}}$. In all cases, the slope of $\log \left(1 / R_{\mathrm{ct}}\right) v s$. potential curves are very similar to Tafel plots determined from the steady-state current density (see Figure 9). The $C_{\text {eff }}$ values of both Ni thin films and NW arrays varied the same way with potential (see below), indicating it reflects an intrinsic surface property. At any fixed potential, lower $R_{\mathrm{ct}}$ and larger $C_{\mathrm{eff}}$ values were obtained for long Ni NWs, as compared to short NW and electrodeposited Ni film (Table 3). Figure 11A and $11 \mathrm{~B}$ shows $C_{\text {eff }}$ values determined at $1.49 \mathrm{~V}$ as a function of the Ni NW length and surface area, respectively. The proportional relationship between $C_{\text {eff }}$ and either NW length or NW surface area confirms that the accessibility of the electrolyte along its axis in not restricted. These results are in close agreement with those discussed previously relating to the electrochemically active Ni surface area probed by CV (see Figure 7). In Fig. 11B, the slope of the curve, representing the intrinsic capacitance per unit area, is $470 \mu \mathrm{F} \mathrm{cm}{ }^{-2}$. This is close to the $C_{\text {eff }}$ for the flat electrodes $\left(700 \mu \mathrm{F} \mathrm{cm}^{-}\right.$ ${ }^{2}$ ), considering that the nanoscale roughness may be different.

Figure 12A shows the variation of $C_{\text {eff }}$ values as a function of the applied potential for a series of NWs of different lengths. In some cases, duplicate samples were made $(2.5 \mu \mathrm{m}$ and $17 \mu \mathrm{m}$ long NW) and the results are very reproducible. It can be clearly seen that all the Ni electrodes display a $C_{\text {eff }}$ that is decreased slightly as the applied potential increases from $1.49 \mathrm{~V}$ to $1.59 \mathrm{~V}$. Figure 12B shows the variation of effective capacitance on 2.5 and $17 \mu \mathrm{m}$ long Ni NW determined by stepping the potential from 1.49 to $1.59 \mathrm{~V}$ and then from 1.59 to $1.49 \mathrm{~V}$. As expected from the previous results, the effective capacitance decreases slightly as the potential is made more positive (anodic sweep). However, it is found that this decrease of the effective capacitance is totally

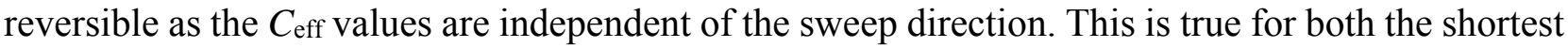
and the longest Ni NWs investigated in the present study, and mirrors the decrease in Ceff seen on the flat surface, (see Figure S6). This strongly suggests that the change in the effective capacitance 
represents a change in the surface condition with potential. This change could be related to a pseudocapacitance of some sort, perhaps associated to the tail end of the oxyhydroxide peak. However, the $\beta-\mathrm{Ni}(\mathrm{OH})_{2} / \beta-\mathrm{NiOOH}$ transition isn't reversible and, unlike what is observed, the $C_{\text {eff }}$ values should be dependent of the sweep direction. As a pseudocapacitance with values roughly as expected for adsorption, this reversible change of $C_{\text {eff }}$ could reflect the potential dependence of an adsorbed species on both Ni thin films and Ni NWs.

The near semicircular low-frequency relaxation exhibiting conventional charge-transfer kinetics and the full accessibility indicated by the proportionality between $C_{\text {eff }}$ and calculated surface area or length raises the question as to whether porous electrode theory is required at all. In its simplest form the theory applies to single isolated electrolyte-filled pores, and then the electrode impedance is determined by taking the parallel impedance of individual pores. In the present case, there is a single interconnected electrolyte volume that seems quite unlike a pore. However, the NW array approximates a periodic 2-D structure in which the impedance of individual 2-D unit cells must be combined in parallel to give the "porous" electrode impedance. We follow a methodology similar to that of Mutha et al. ${ }^{34}$ In the present case, the array is hexagonal with one nanowire per unit cell. The theory for cylindrical pores therefore needs little modification for cylindrical nanowires: the curved surface (wall) area ( $\left.a_{\text {wall }}\right)$ is the same, but the cross sectional area of the electrolyte-filled pore ( $\left.a_{\text {pore }}\right)$ is now the area of the unit cell minus the circular area of the nanowire.

In the present case the unit cell area is the reciprocal of the number density of NWs determined from the SEM images, and the NW area is known from the diameters, also measured from the SEM images. Furthermore, the flat Ni electrode has a 1-CPE interfacial impedance, so that the porous electrode theory gives the same equation as given by Hitz and Lasia, ${ }^{28}$ with the exception that their parameter $a$ is slightly different. In the general case, $a^{-1}=a_{\text {wall }} R_{\text {pore }}$ and $R_{\text {pore }}=\rho l / n a_{\text {pore }}$ 
where $l$ is the pore length and $\rho$ is the resistivity of the electrolyte, and only $a_{\text {pore }}$ is different from the cylindrical pore case. With the known geometric factors and appropriate kinetic parameters, the low frequency semicircle shape and size are well predicted, but the high-frequency feature should have size of the order of $R_{\text {pore }}$, which is much smaller than observed.

The key parameter determining the shape is the penetration depth of the a.c. signal, $\lambda=$ $\left(a_{\mathrm{pore}} \mid Z_{\mathrm{eq}} / \rho \pi d\right)^{1 / 2}$, where $d$ is the diameter of the nanowires and $Z_{\text {eq }}$ is the specific $\left.(\mathrm{Ohm} \mathrm{cm})^{2}\right)$ interfacial impedance of the pore walls on a real area basis. $Z_{e q}$ was calculated as a parallel combination of a charge transfer resistance and $\mathrm{CPE}$, with values taken from the fitted values for the low-frequency semicircle and scaled by the roughness factor to convert to a real area basis. This depth is independent of pore/wire length and decreases from $540 \mu \mathrm{m}$ at $0 \mathrm{~Hz}$ to $24 \mu \mathrm{m}$ at $100 \mathrm{~Hz}$ for the kinetic parameters at $1.59 \mathrm{~V}$. It will be larger for more negative potentials, and we conclude that for the frequencies relevant for the low-frequency feature, it is always much larger than the wire length. Therefore, the pore is fully accessible and the kinetics solely determine the shape. This justifies the simplified analysis above that neglected the details of the porous electrode theory.

The same analysis predicts a high-frequency part that is a $45^{\circ}$ line and not a semicircle. Such $45^{\circ}$ lines have been observed for ideal pore geometries such as macroscopic micro-wire brush electrodes ${ }^{35}$ or wire-in-capillary electrodes ${ }^{36}$ However, the shape of the high-frequency feature is known to depend on the pore shape, with semicircles predicted theoretically for pear-shaped pores ${ }^{37}$ and observed experimentally for pressed powder porous Ni electrodes. ${ }^{28}$ The present geometry seems far from these. However, as shown in Figure S7, the shape of the high-frequency part of the electrochemical impedance spectra evolves with the NW length, and thus the pore aspect ratio since the NW-NW distance is fixed at ca $80 \mathrm{~nm}$ in the present study. This is reminiscent of the findings of Hitz and Lasia, who showed that the high frequency feature of spherical pores becomes flatter 
and more linear as the pore openings becomes larger. ${ }^{28}$ This issue will need to be more closely investigated in the future.

\section{Conclusion}

Long Ni NWs were synthesized by electrodeposition through an AAO membrane. The Ni NWs have a diameter of ca $300 \mathrm{~nm}$. Detailed structural analysis showed that each Ni NWs is composed of a central Ni metallic core and surrounded by a $\mathrm{Ni}(\mathrm{OH})_{2}$ crust that thickens from $10 \mathrm{~nm}$ to $20 \mathrm{~nm}$ after extensive electrochemical testing. The 1D Ni nanowires are densely packed in a brush-like array structure, with a packing density between that expected for ideal square- and hexagonal-packing. The NW-NW distance was kept constant at $c a .80 \mathrm{~nm}$ and thus the aspect ratio of the electrolyte-filled volume between pairs of NWs is decreased from $2.7 \%$ to $0.4 \%$ as the NW length is increased by one order of magnitude up to $20 \mu \mathrm{m}$. A proportional relationship is observed between the electrochemically active Ni surface area probed by CV and either NW length or NW surface area, confirming that the accessibility of the electrolyte along its axis in not restricted despite the huge restriction at the openings of the electrolyte-filled volume. Likewise, the proportionality between $C_{\text {eff }}$ and calculated NW surface area suggests that the accessibility of the electrolyte along the NW axis is not restricted. All data points to the OER being kinetically-controlled in the overpotential range investigated here, with very little (if any) mass-transport limitation of dissolved oxygen, ionic migration within the pores, or bubble occlusion effects. The governing factor affecting the electrocatalytic properties of closely packed $20 \mu \mathrm{m}$ long Ni NW arrays for the OER is the enhanced Ni surface area for the ordered 1D nanostructures, which translates into a significant 200 $\mathrm{mV}$ decrease of the OER overpotential at $10 \mathrm{~mA} \mathrm{~cm}^{-2}$. These vertically aligned 1D NWs allow to increase the electrochemically active surface area of a Ni plate by a factor of 130 without introducing 
any limitation by diffusion. This can be due to fact that the 1D NWs are almost perfectly aligned. It is expected that they could serve as an ideal platform to support better catalytic materials without showing any limitation due to mass-transport.

\section{Supporting Information}

Fig. S1 SEM images illustrating the stage-by-stage lifting out of the TEM specimen using focussed ion beam milling.

Fig. S2 Estimation of packing fraction in Ni-NWs with segmentation analyses of the SEM images.

Fig. S3 SEM micrographs for Ni NW arrays with different NW lengths.

Fig. S4 Spatially resolved Maps and Line Profiles of the O-K edge in the case of pristine Ni NWs and Ni NWs post-treatment in 1M KOH.

Table S1 Fitting parameters of different electrodes obtained by using the corresponding equivalent circuits shown in Figure 10.

Fig. S5 Product of Steady State Current Density and $R_{p}=R_{1}+R_{c t}$ as a function of potentials on $17 \mu \mathrm{m}$ Ni NWs.

Fig. S6 Normalized effective capacitance in function of potential.

Fig. S7 High-frequency part of the IES spectra recorded at $1.49 \mathrm{~V}$ vs RHE.

\section{Acknowledgement}

This research was conducted as part of the Engineered Nickel Catalysts for Electrochemical Clean Energy project administered from Queen's University and supported by Grant No. RGPNM 477963-2015 under the Natural Sciences and Engineering Research Council of Canada (NSERC) 
Discovery Frontiers Program. Part of the electron microscopy work was carried out at the Canadian Centre for Electron Microscopy, a national facility supported by the Canada Foundation of Innovation under the MSI program, NSERC, and McMaster University. 


\section{Captions}

Figure 1 High-resolution SEM images of the Ni NWs synthesized revealing their crosssectional and top- views. (a) Low-magnification SEM image. (b) High-resolution SEM image. (c) Cross-sectional view of the NW structure.

Figure 2 SEM top-view micrographs of (a) as-received AAO membrane; (b) Ni deposited in AAO membrane before the later was removed; (c) Ni NWs arrays just after the AAO membrane was dissolved: and (d) Ni NWs after extensive electrochemical characterization. Inset Figures - Corresponding holes and NW diameter size distribution plots.

Figure 3 High-resolution SEM images comparing the morphology of pristine Ni NWs and those post electrochemical treatment under alkaline media.

Figure 4 Compositional analyses of pristine Ni NWs with EELS. (a) Cross-sectional SEM view. (b), (c), and (d), Elemental Mapping of Ni, O and Ti over the bottom, middle and top regions of a typical Ni NW.

Figure 5 Detailed analyses of the $\mathrm{Ni}_{2} \mathrm{~L}_{3} / \mathrm{L}_{2}$ white-line intensity ratios to deduce the chemical state of Ni in the Pristine (a) and electrochemically treated (b) Ni NWs. (ii) - Spectrum extracted over the core region. (iii) - Spectrum extracted over the shell region.

Figure 6 Stable $\mathrm{CV}\left(25 \mathrm{mV} \mathrm{s}^{-1}\right)$ for electrodeposited Ni thin film in $1 \mathrm{M} \mathrm{KOH}$. In (A), the upper potential limit is restricted to $0.45 \mathrm{~V}$ to prevent the formation of $\beta-\mathrm{Ni}(\mathrm{OH})_{2}$. In (B), the positive potential limit of CVs was extended.

Figure 7 Variation of $Q_{a}$ with respect to the measured Ni NW lengths. $Q_{a}$ values were determined from $\mathrm{CV}$ s recorded between in -0.10 and $0.45 \mathrm{~V}$ in $1 \mathrm{M} \mathrm{KOH}$ at $25 \mathrm{mV} \mathrm{s}^{-}$

1. The dotted red line corresponds to the charge expected based on a theoretical 
calculation assuming each NW is a perfect cylinder of known diameter and length, and assuming the specific charge density for the conversion of $\mathrm{Ni}$ to $\alpha-\mathrm{Ni}(\mathrm{OH})_{2}$ is 0.514 $\mathrm{mC} \mathrm{cm}{ }^{-2}$.

Figure 8 In (A), chronopotentiometric curves recorded at $10 \mathrm{~mA} \mathrm{~cm}^{-2}$ on different Ni samples. The electrolyte was $1 \mathrm{M} \mathrm{KOH}$. The potential was corrected for the ohmic drop. In (B), influence of $Q_{\mathrm{a}}$ on the OER stability in $1 \mathrm{M} \mathrm{KOH}$ at $j=10 \mathrm{~mA} \mathrm{~cm}^{-2}$. The $i R$-corrected potential after 1 and 30 minutes of electrolysis are plotted. In (C), iR-corrected potential after 30 minutes of electrolysis as a function of $Q_{a}$.

Figure 9 Variation of steady-state current density, $j,(\mathrm{~A} 1, \mathrm{~B} 1$ and $\mathrm{C} 1)$ and $\log \left(1 / R_{c t}\right)(\mathrm{A} 2, \mathrm{~B} 2$ and $\mathrm{C} 2$ ) with respect to $i R$-corrected potential. The data are shown for electrodeposited Ni thin film (A1 and A2), $3 \mu \mathrm{m}$ NW electrode (B1 and B2) and $17 \mu \mathrm{m} \mathrm{NW}$ electrode (C1 and $\mathrm{C} 2)$.

Figure 10 Measured (solid lines) and fitted (symbols) EIS plots measured on a) electrodeposited Ni thin film; b) $2.5 \mu \mathrm{m}$ Ni NW electrode; c) $17 \mu \mathrm{m} \mathrm{Ni} \mathrm{NW} \mathrm{electrode} \mathrm{at} \mathrm{different}$ potentials. The equivalent electrical circuits used to fit the experimental data are also shown.

Figure 11 The effective capacitance in function of a) NW length and b) NW calculated surface area.

Figure 12 In (A), effective capacitance in function of potential on different $\mathrm{Ni}$ electrodes including duplicate experiments. In (B), effective capacitance in function of potential on $2.5 \mu \mathrm{m}$ and $17 \mu \mathrm{m}$ long Ni NW electrodes tested for increasing and then decreasing potentials. 
Table 1: Quantification of Elemental Edges in EELS for the Pristine Ni NW sample.

\begin{tabular}{|c|c|c|c|}
\hline $\begin{array}{c}\text { EELS Elemental } \\
\text { Quantification }\end{array}$ & Ti & O & Ni \\
\hline Middle Region & $<1$ at. $\%$ & 18.3 at. $\%$ & 81.7 at. $\%$ \\
\hline Bottom Region & 8.1 at. $\%$ & 29.4 at. $\%$ & 62.5 at. $\%$ \\
\hline
\end{tabular}


Table 2: Comparison of $\mathrm{L}_{3} / \mathrm{L}_{2}$ white-line intensity ratios of pristine and post-treated Ni NWs with the $\mathrm{NiO}_{2}, \mathrm{Ni}(\mathrm{OH})_{2}$ and $\mathrm{Ni}$ references.

\begin{tabular}{|c|c|c|}
\hline Estimated L3/L2 Ratios & Core Region & Shell Region \\
\hline Pristine Ni NWs & 3.38 & 3.63 \\
\hline Ni NWs Post-treatment & 3.41 & 3.56 \\
\hline Ni Reference & \multicolumn{2}{|c|}{$3.3[1]$} \\
\hline NiO Reference & \multicolumn{2}{|c|}{$3.8[2] ; 4.0[3]$} \\
\hline Ni(OH)2 Reference & \multicolumn{2}{|c|}{$3.6[2]$} \\
\hline
\end{tabular}

[1] P. A. Van Aken et al., Phys. Chem. Minerals, 29 (2002) 188.

[2] From the estimation of $\mathrm{Ni}-\mathrm{L}_{2,3}$ Edges in pure $\mathrm{NiO}_{2}$ and $\mathrm{Ni}(\mathrm{OH})_{2}$ samples obtained from Sigma Aldrich.

[3] R. D. Leapman, L. A. Grunes, \& P. L. Fejes, Study of the L2,3 edges in the 3 d transition metals and their oxides by electron-energy-loss spectroscopy with comparisons to theory. Physical Review B, 26 (1982) 614. 
Table 3: Fitted results from EIS analyses, and equivalent circuits displayed in Fig. 10.

\begin{tabular}{|c|c|c|c|}
\hline Sample & $\begin{array}{c}E_{\text {appl }} / \\
\text { V vs RHE } \\
\end{array}$ & $\begin{array}{c}R_{\mathrm{ct}} / \\
\Omega \mathrm{cm}_{\text {geo }}^{2}\end{array}$ & 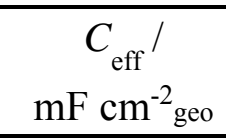 \\
\hline \multirow{6}{*}{$\begin{array}{l}\text { Electrodeposited } \\
\text { Ni film }\end{array}$} & 1.49 & $1979 \pm 94$ & $0.72 \pm 0.02$ \\
\hline & 1.51 & $736 \pm 17$ & $0.65 \pm 0.02$ \\
\hline & 1.53 & $307 \pm 4$ & $0.60 \pm 0.02$ \\
\hline & 1.55 & $148 \pm 1$ & $0.56 \pm 0.02$ \\
\hline & 1.57 & $84.2 \pm 0.5$ & $0.52 \pm 0.01$ \\
\hline & 1.59 & $56.1 \pm 0.3$ & $0.47 \pm 0.02$ \\
\hline \multirow{6}{*}{$\begin{array}{c}3 \mu \mathrm{m} \\
\text { Ni nanowires }\end{array}$} & 1.49 & $931 \pm 36$ & $8.50 \pm 0.08$ \\
\hline & 1.51 & $443 \pm 7$ & $7.74 \pm 0.05$ \\
\hline & 1.53 & $204 \pm 2$ & $7.33 \pm 0.05$ \\
\hline & 1.55 & $105.4 \pm 0.6$ & $7.02 \pm 0.07$ \\
\hline & 1.57 & $61.7 \pm 0.4$ & $6.85 \pm 0.10$ \\
\hline & 1.59 & $40.2 \pm 0.2$ & $6.62 \pm 0.10$ \\
\hline \multirow{6}{*}{$\begin{array}{c}17 \mu \mathrm{m} \\
\text { Ni nanowires }\end{array}$} & 1.49 & $316 \pm 30$ & $58.16 \pm 0.21$ \\
\hline & 1.51 & $180 \pm 8$ & $52.30 \pm 0.22$ \\
\hline & 1.53 & $96 \pm 2$ & $47.93 \pm 0.23$ \\
\hline & 1.55 & $58 \pm 1$ & $41.71 \pm 0.24$ \\
\hline & 1.57 & $35.8 \pm 0.3$ & $41.86 \pm 0.21$ \\
\hline & 1.59 & $24.6 \pm 0.2$ & $43.14 \pm 0.30$ \\
\hline
\end{tabular}




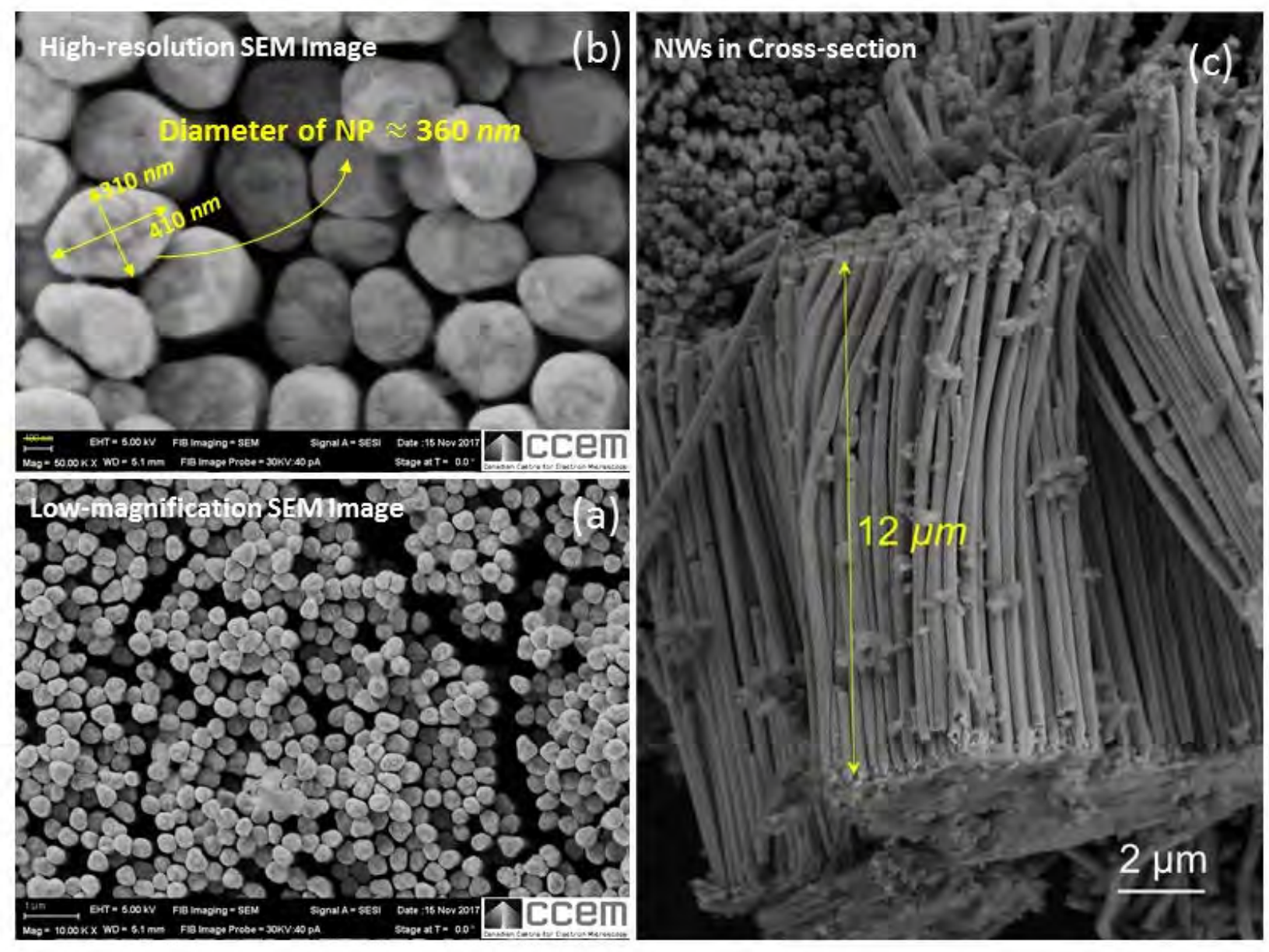

Figure 1 

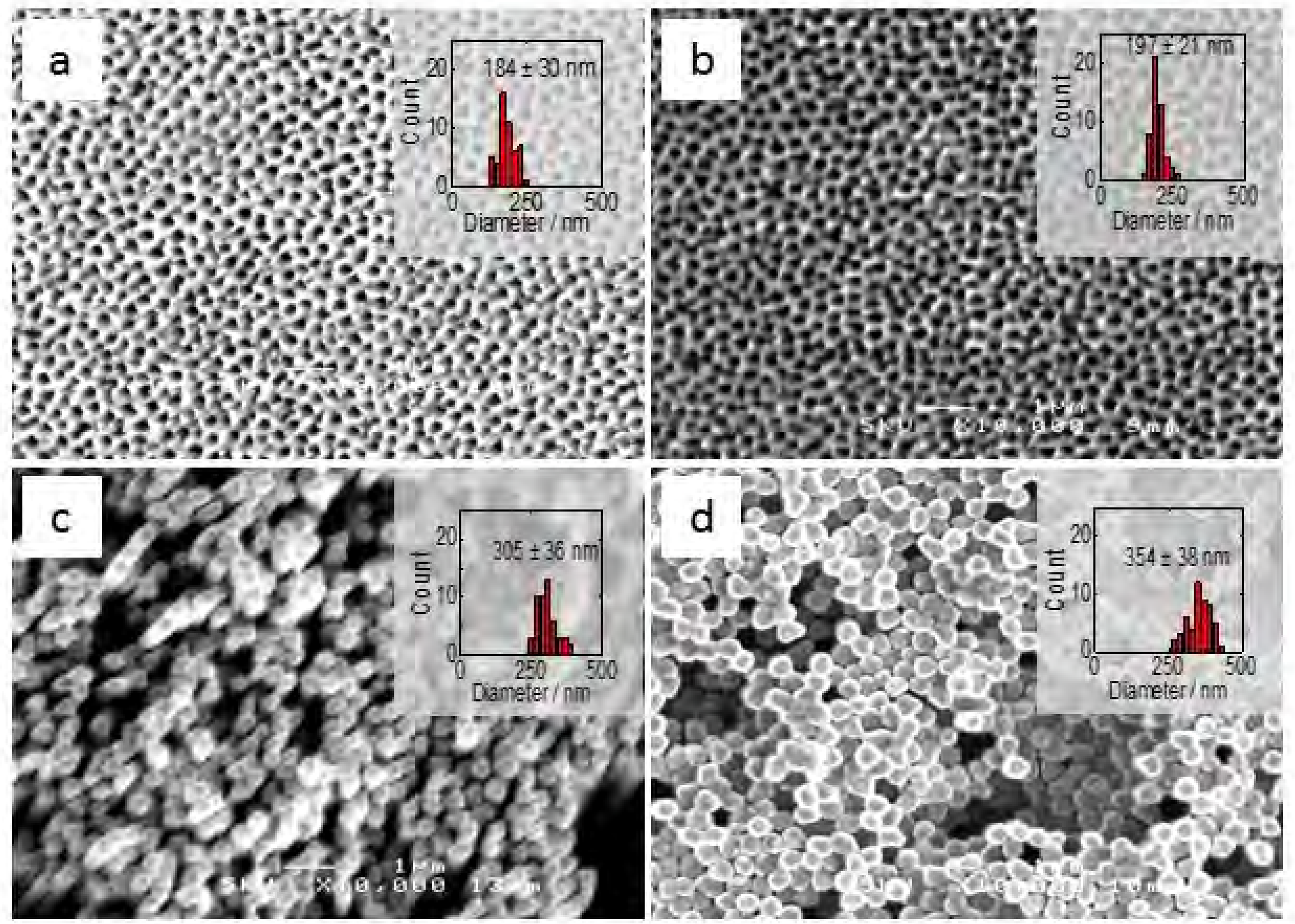

Figure 2 


\section{Pristine Ni NWs}

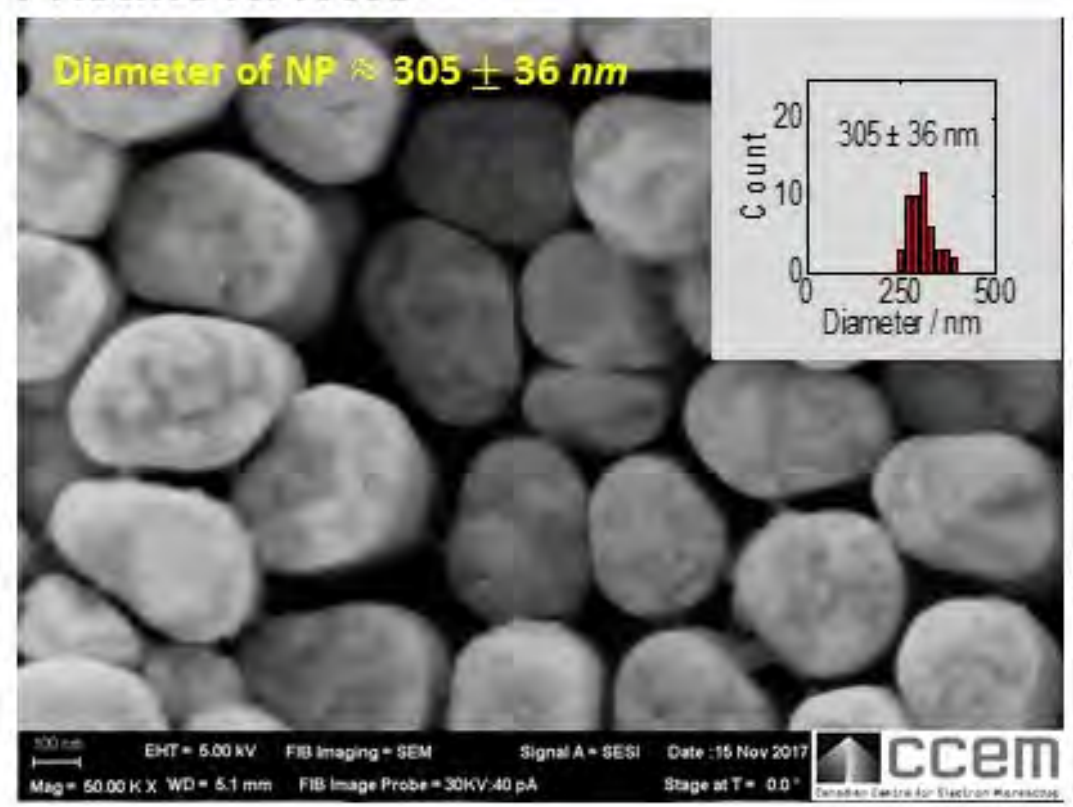

Ni NWs Post-treatment in $1 \mathrm{M} \mathrm{KOH}$

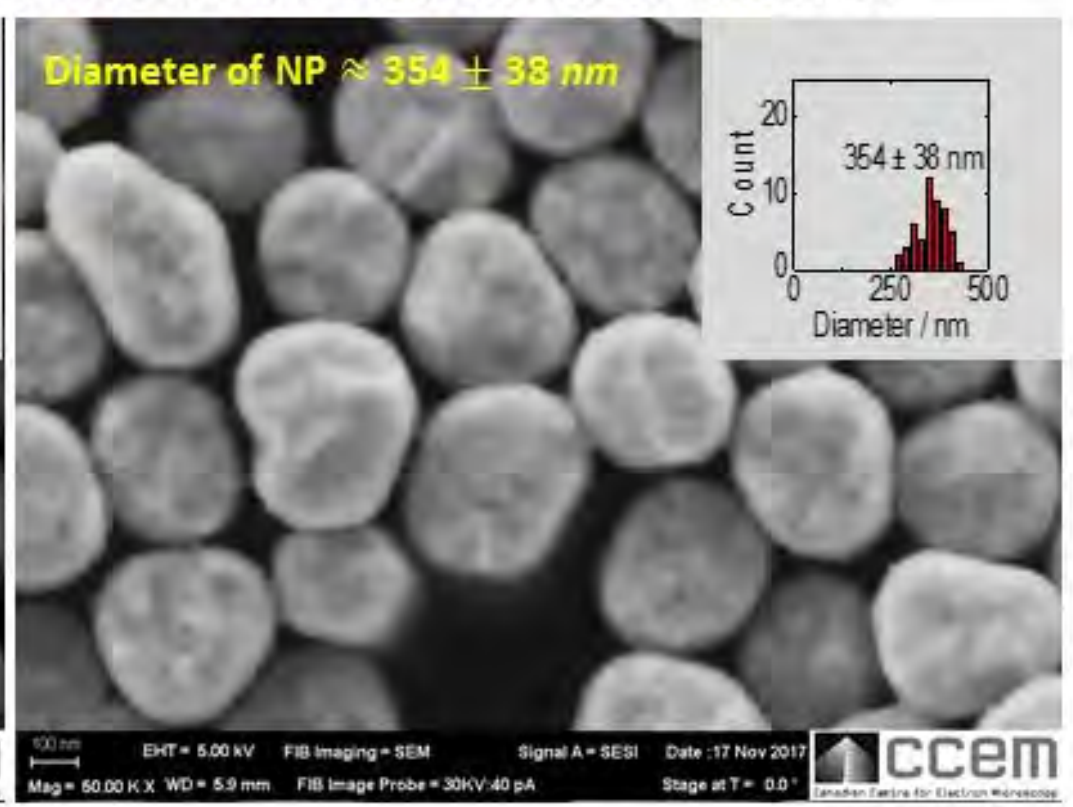

Figure 3 

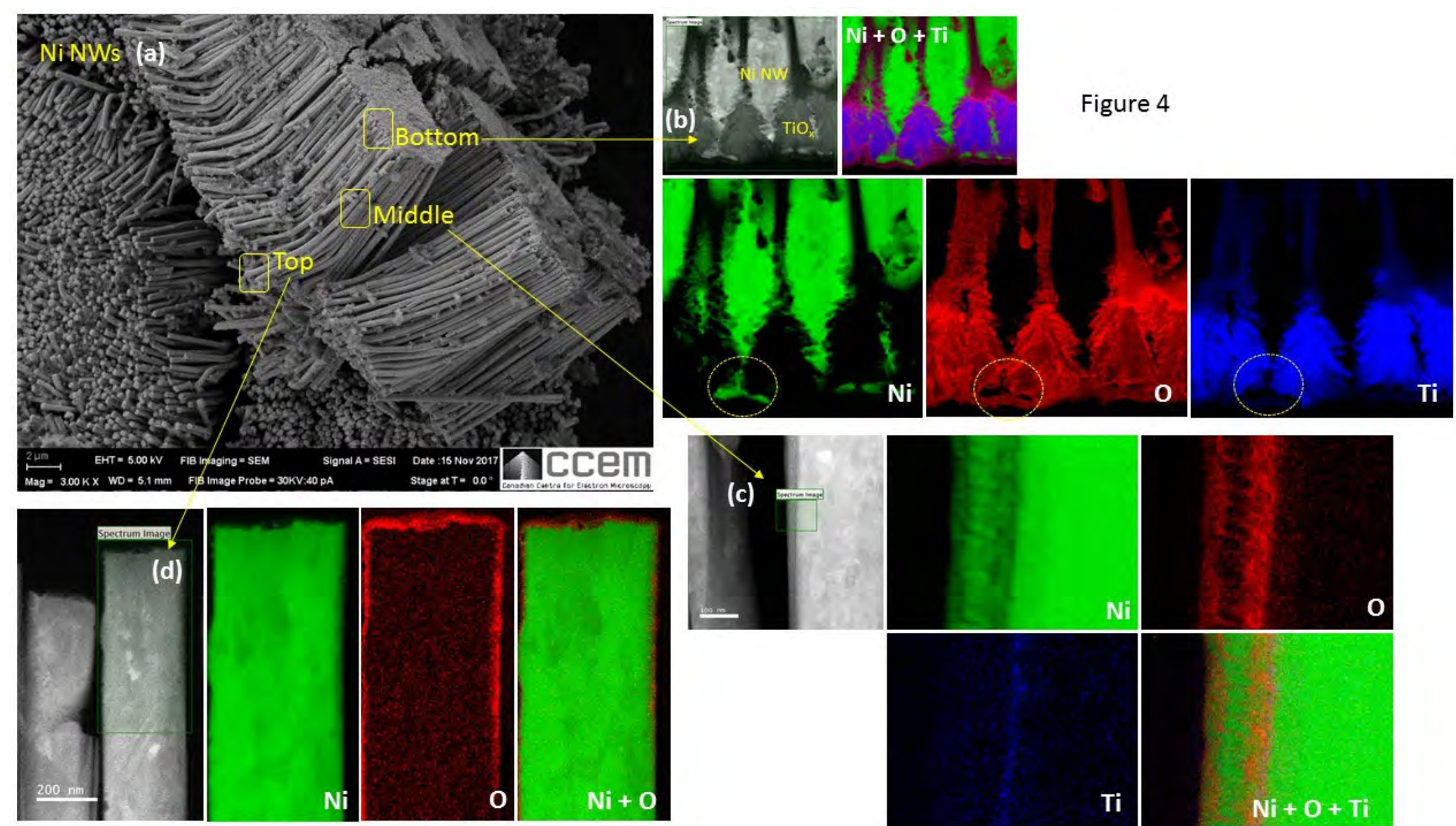

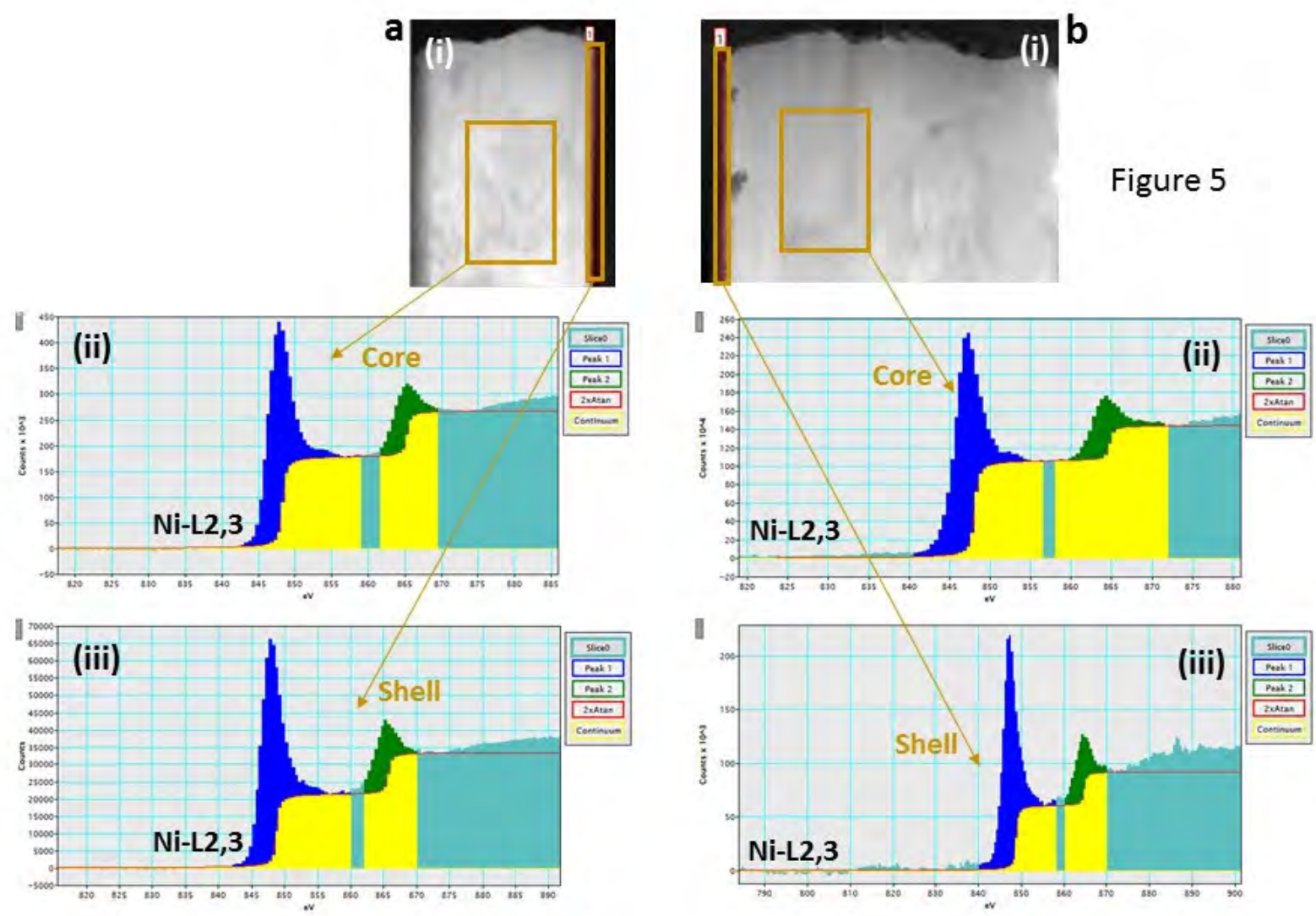

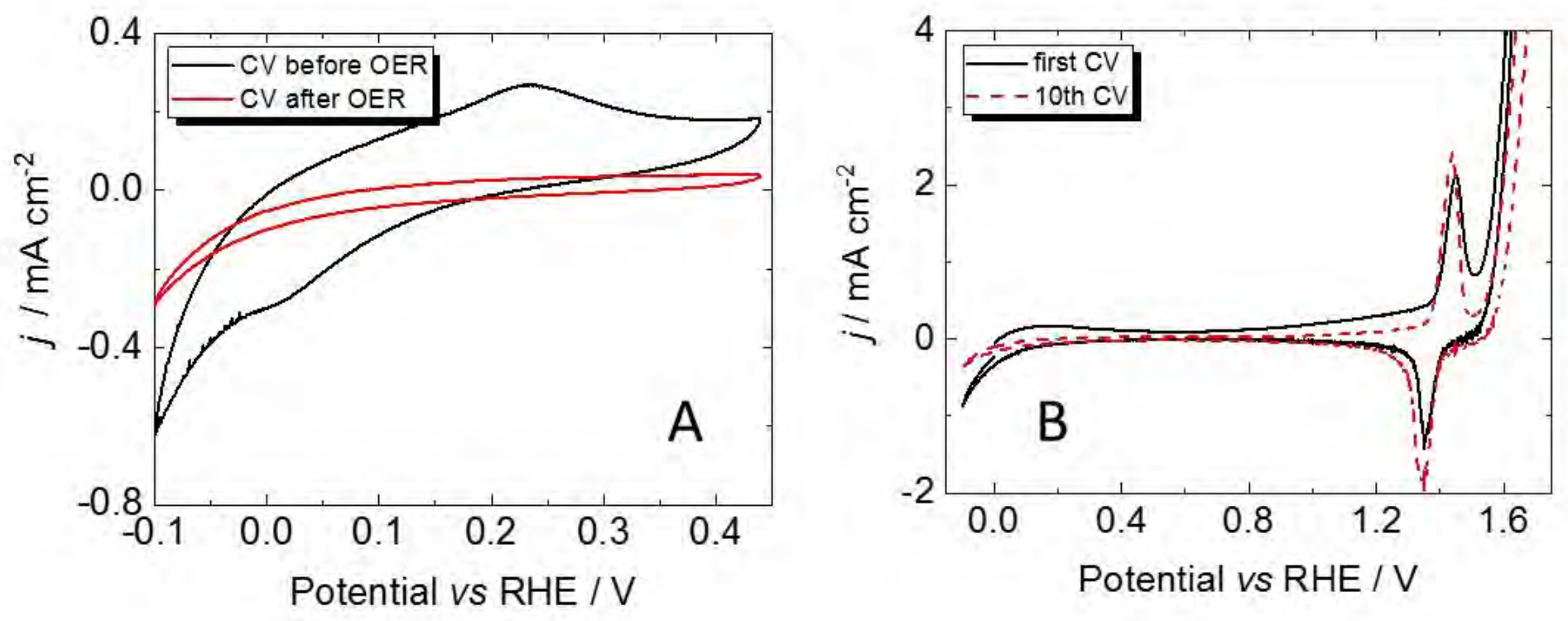

Figure 6 


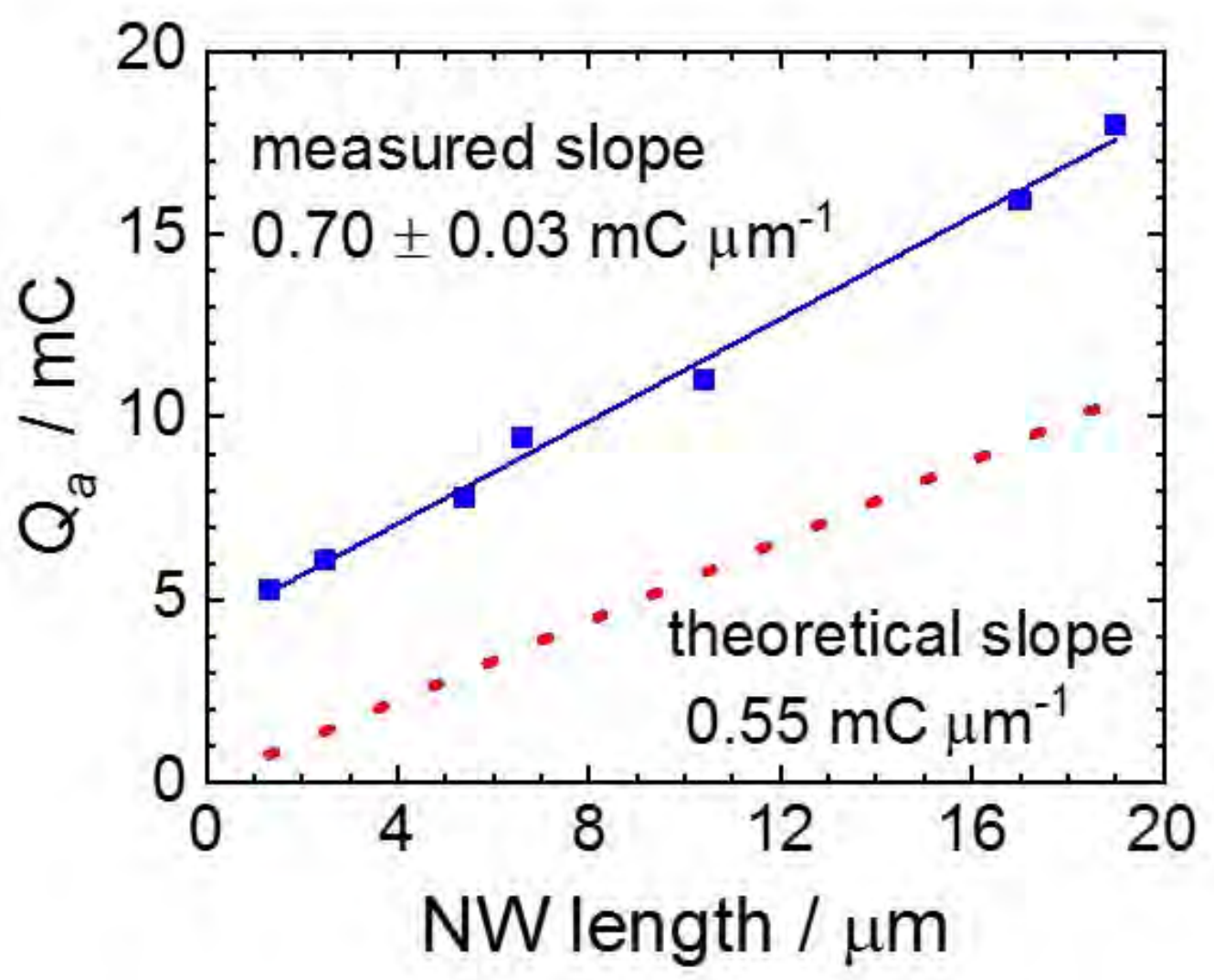

Figure 7 

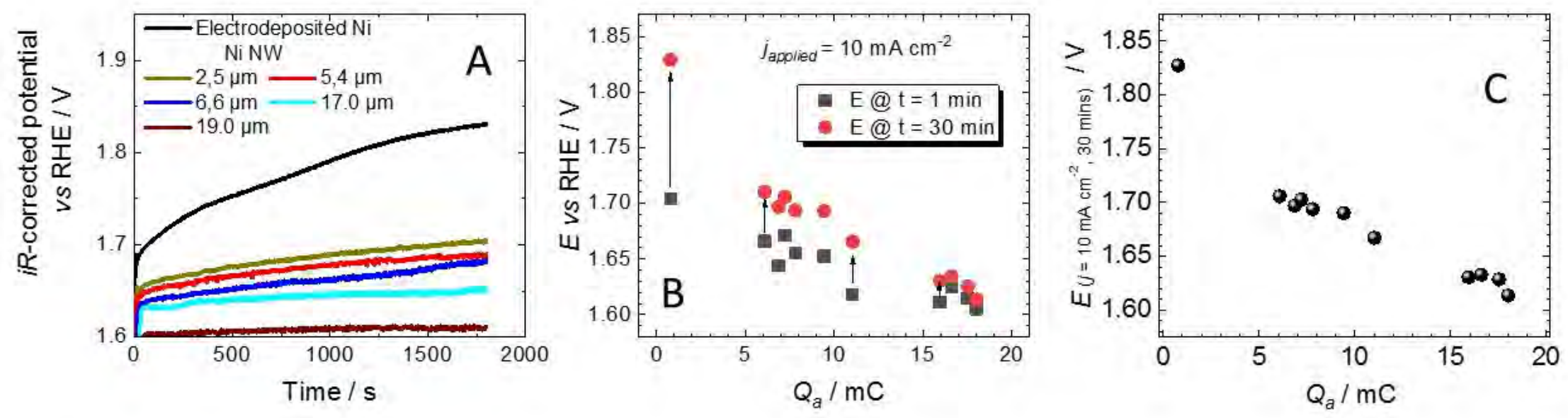

Figure 8 

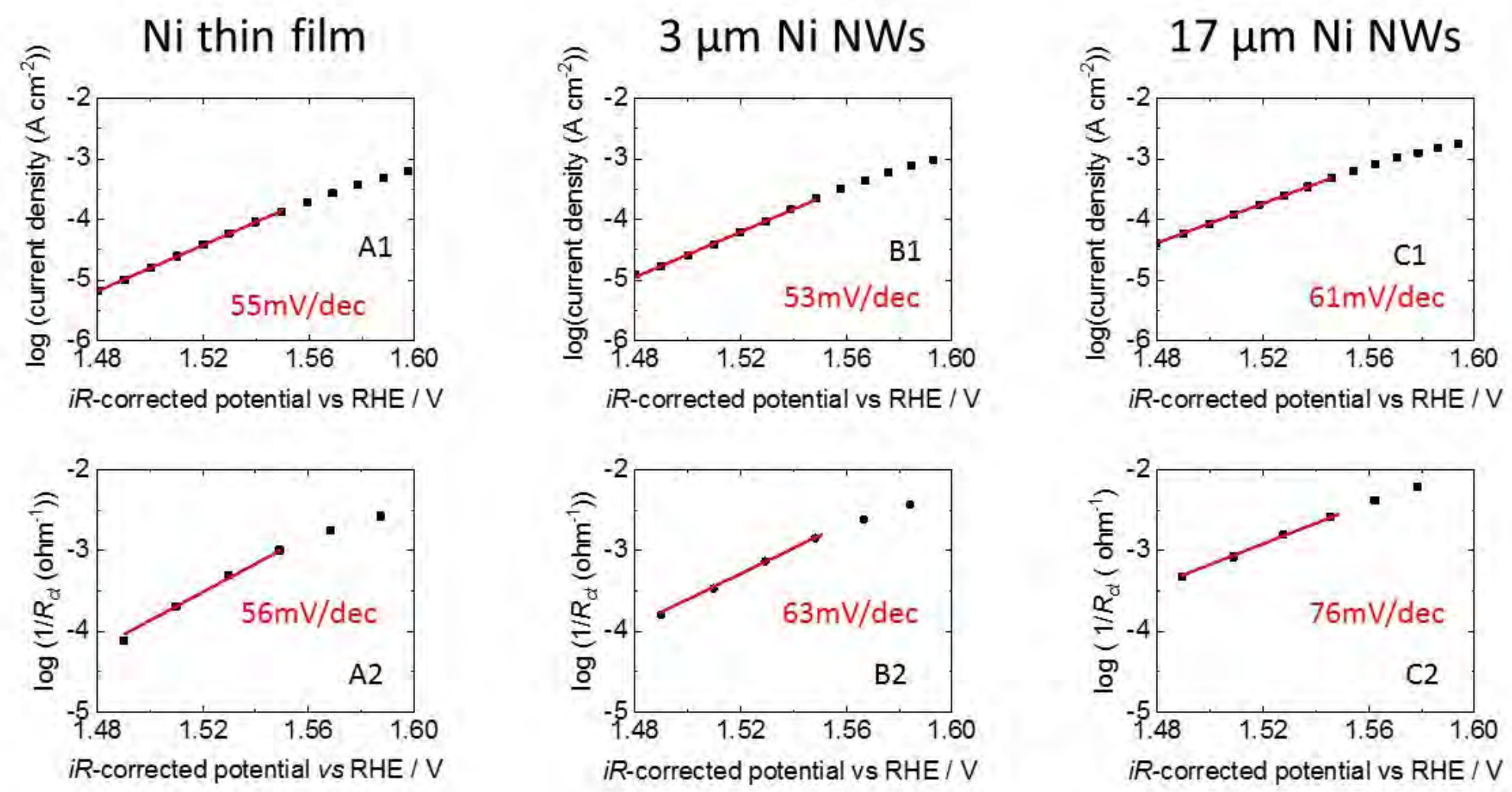

Figure 9 

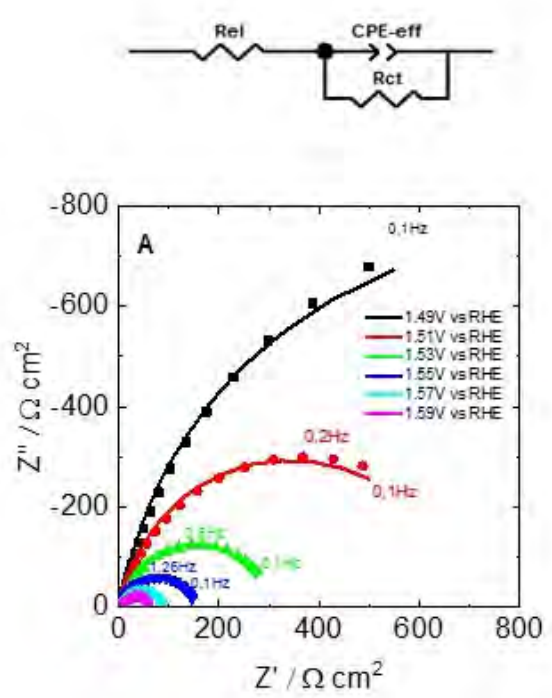
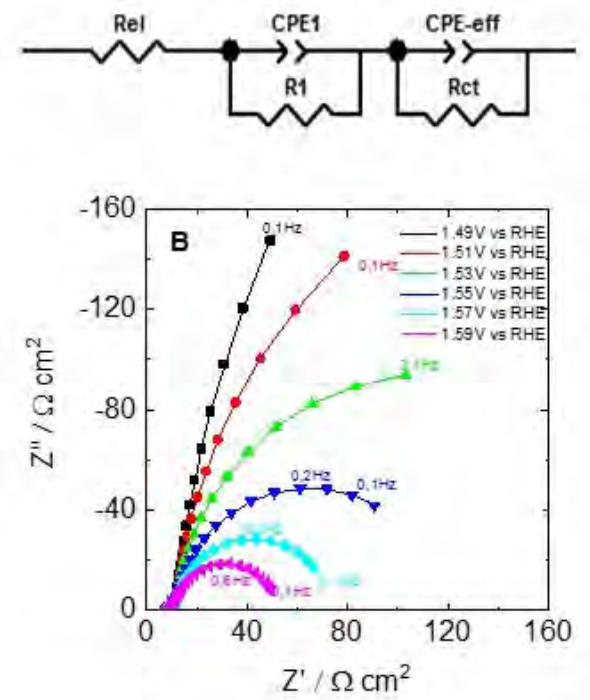
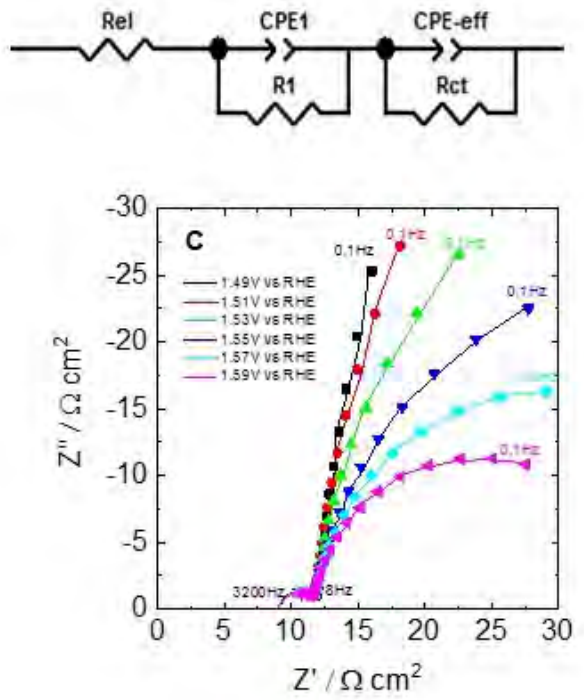

Figure 10 

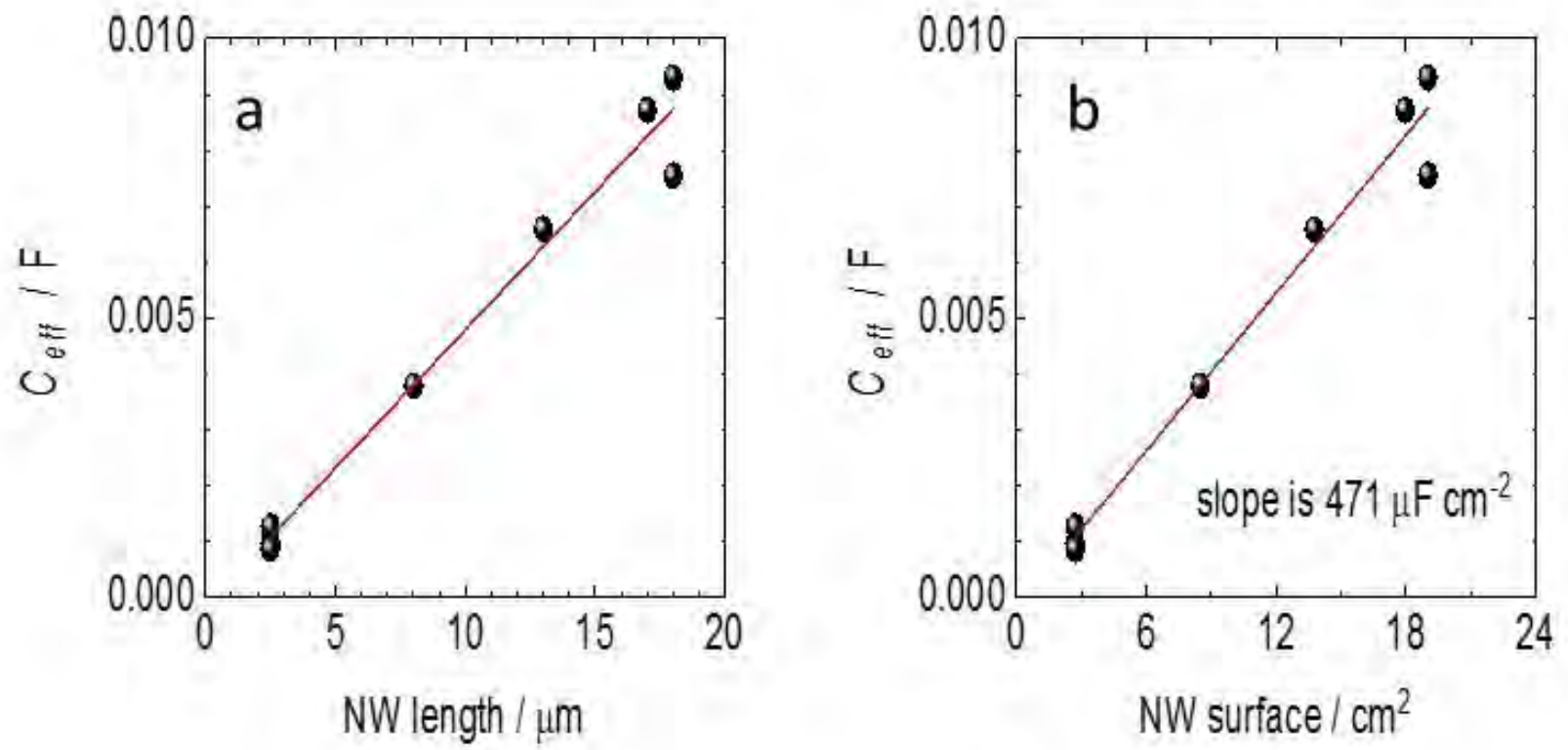

Figure 11 


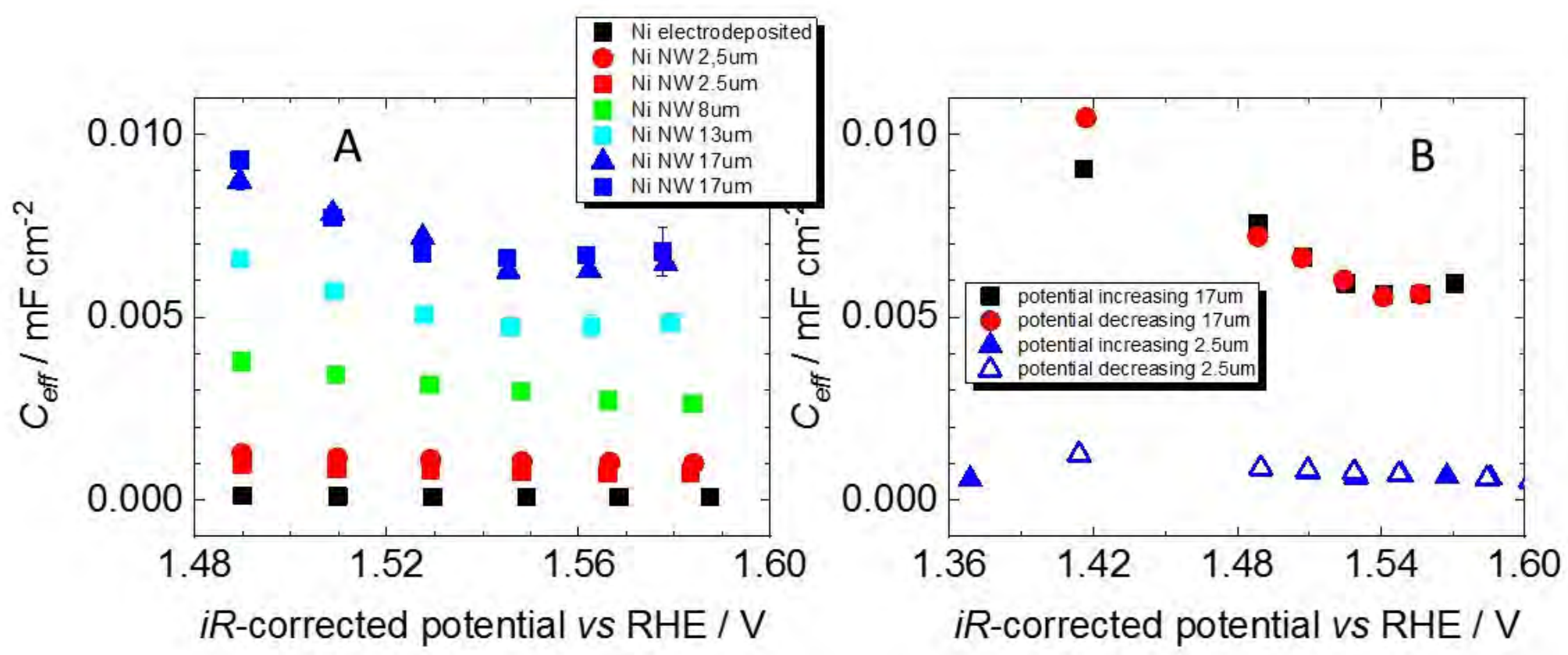

Figure 12 


\section{References}

1 Hickner, M. A. Strategies for Developing New Anion Exchange Membranes and Electrode Ionomers. Electrochem. Soc. Interface 2017, 26, 69-73.

2 Hagesteijn, K. F. L.; Jiang, S.; Ladewig, B. P. A Review of the Synthesis and Characterization of Anion Exchange Membranes. J. Mater. Sci. 2018, 53, 11131-11150.

3 Zheng, Y.; Ash, U.; Pandey, R. P.; Ozioko, A. G.; Ponce-González, J.; Handl, M.; Weissbach, T.; Varcoe, J. R.; Holdcroft, S.; Liberatore, M. W.; et al. Water Uptake Study of Anion Exchange Membranes. Macromolecules 2018, 51, 3264-3278.

4 Schibli, E. M.; Wright, A. G.; Holdcroft, S.; Frisken, B. J. Morphology of Anion-Conducting Ionenes Investigated by X-Ray Scattering and Simulation. J. Phys. Chem. B 2018, 122, $1730-1737$.

5 Luo, X.; Wright, A.; Weissbach, T.; Holdcroft, S. Water Permeation through Anion Exchange Membranes. J. Power Sources 2018, 375, 442-451.

6 Corrigan, D. A.; Soc, J. E.; Corrigan, D. A. The Catalysis of the Oxygen Evolution Reaction by Iron Impurities in Thin Film Nickel Oxide Electrodes The Catalysis of the Oxygen Evolution Reaction by Iron Impurities in Thin Film Nickel Oxide Electrodes. J. Electrochem. Soc. $1987,134,377-384$.

7 Trotochaud, L.; Young, S. L.; Ranney, J. K.; Boettcher, S. W. Nickel - Iron Oxyhydroxide Oxygen-Evolution Electrocatalysts: The Role of Intentional and Incidental Iron Incorporation. J. Am. Chem. Soc. 2014, 136, 6744-6753. 
8 Lu, F.; Zhou, M.; Zhou, Y.; Zeng, X. First-Row Transition Metal Based Catalysts for the Oxygen Evolution Reaction under Alkaline Conditions: Basic Principles and Recent Advances. Small 2017, 13, 1701931.

9 Suen, N.-T.; Hung, S.-F.; Quan, Q.; Zhang, N.; Xu, Y.-J.; Chen, H. M. Electrocatalysis for the Oxygen Evolution Reaction: Recent Development and Future Perspectives. Chem. Soc. Rev. 2017, 46, 337-365.

10 Kim, J. S.; Kim, B.; Kim, H.; Kang, K. Recent Progress on Multimetal Oxide Catalysts for the Oxygen Evolution Reaction. Adv. Energy Mater. 2018, 8, 1702774.

11 Feng, G.; Kuang, Y.; Li, Y.; Sun, X. Three-Dimensional Porous Superaerophobic Nickel Nanoflower Electrodes for High-Performance Hydrazine Oxidation. Nano Res. 2015, 8, $3365-3371$.

12 Lu, Z.; Zhu, W.; Yu, X.; Zhang, H.; Li, Y.; Sun, X.; Wang, X.; Wang, H.; Wang, J.; Luo, J.; et al. Ultrahigh Hydrogen Evolution Performance of Under-Water "Superaerophobic" MoS 2 Nanostructured Electrodes. Adv. Mater. 2014, 26, 2683-2687.

13 Zhang, Q.; Li, P.; Zhou, D.; Chang, Z.; Kuang, Y.; Sun, X. Superaerophobic Ultrathin NiMo Alloy Nanosheet Array from In Situ Topotactic Reduction for Hydrogen Evolution Reaction. Small 2017, 13, 1701648.

14 He, J.; Hu, B.; Zhao, Y. Superaerophobic Electrode with Metal@Metal-Oxide Powder Catalyst for Oxygen Evolution Reaction. Adv. Funct. Mater. 2016, 26, 5998-6004.

15 Li, Y.; Zhang, H.; Jiang, M.; Kuang, Y.; Sun, X.; Duan, X. Ternary NiCoP Nanosheet Arrays: An Excellent Bifunctional Catalyst for Alkaline Overall Water Splitting. Nano Res. 2016, 9, 2251-2259. 
16 Faber, M. S.; Dziedzic, R.; Lukowski, M. A.; Kaiser, N. S.; Ding, Q.; Jin, S. HighPerformance Electrocatalysis Using Metallic Cobalt Pyrite ( $\mathrm{CoS}_{2}$ ) Micro- and Nanostructures. J. Am. Chem. Soc. 2014, 136, 10053-10061.

17 Li, Y.; Zhang, H.; Xu, T.; Lu, Z.; Wu, X.; Wan, P.; Sun, X.; Jiang, L. Under-Water Superaerophobic Pine-Shaped Pt Nanoarray Electrode for Ultrahigh-Performance Hydrogen Evolution. Adv. Funct. Mater. 2015, 25, 1737-1744.

18 Van Aken, P. A.; Liebscher, B. Quantification of Ferrous/Ferric Ratios in Minerals: New Evaluation Schemes of Fe L 23 Electron Energy-Loss near-Edge Spectra. Phys. Chem. Miner. 2002, 29, 188-200.

19 Martin, M. H.; Galipaud, J.; Tranchot, A.; Roué, L.; Guay, D. Measurements of Hydrogen Solubility in $\mathrm{Cu}_{\mathrm{x}} \mathrm{Pd}_{100-\mathrm{x}}$ thin Films. Electrochim. Acta 2013, 90, 615-622.

20 Alsabet, M.; Grden, M.; Jerkiewicz, G. Electrochemical Growth of Surface Oxides on Nickel . Part 1 : Formation of $\alpha-\mathrm{Ni}(\mathrm{OH})_{2}$ in Relation to the Polarization Potential, Polarization Time, and Temperature. Electrocatal 2011, 2, 317-330.

21 Scarr, R. F. The Mechanism of Oxygen Evolution on Nickel, Platinum, and Other Metals and Alloys. J. Electrochem. Soc. 1969, 116, 1526.

22 Gennero de Chialvo, M. R.; Chialvo, A. C. Oxygen Evolution Reaction on Thick Hydrous Nickel Oxide Electrodes. Electrochim. Acta 1988, 33, 825-830.

23 Lyons, M. E. G.; Brandon, M. P. A Comparative Study of the Oxygen Evolution Reaction on Oxidised Nickel, Cobalt and Iron Electrodes in Base. J. Electroanal. Chem. 2010, 641, $119-130$. 
24 Zhou, T.; Cao, Z.; Zhang, P.; Ma, H.; Gao, Z.; Wang, H.; Lu, Y.; He, J.; Zhao, Y. Transition Metal Ions Regulated Oxygen Evolution Reaction Performance of Ni-Based Hydroxides Hierarchical Nanoarrays. Sci. Rep. 2017, 7, 46154.

25 De Levie, R. Electrochemical response of porous and rough electrodes. In Advances in Electrochemistry and Electrochemical Engineering; Delahay, P., Ed.; Willey-Interscience: New York, 1967; Vol. 6, pp 329-397.

26 Lasia, A. Electrochemical Impedance Spectroscopy and Its Applications; Springer: New York, 2014.

27 Orazem, M. E.; Tribollet, B. Electrochemical Impedance Spectroscopy; Wiley: Hoboken, NJ, 2008.

28 Hitz, C.; Lasia, A. Experimental Study and Modeling of Impedance of the Her on Porous Ni Electrodes. J. Electroanal. Chem. 2001, 500, 213-222.

29 Seland, F.; Tunold, R.; Harrington, D. A. Impedance Study of Methanol Oxidation on Platinum Electrodes. Electrochim. Acta 2006, 51, 3827-3840.

30 Ramulu, T. S.; Venu, R.; Sinha, B.; Lim, B.; Jeon, S. J.; Yoon, S. S.; Kim, C. G. Nanowires Array Modified Electrode for Enhanced Electrochemical Detection of Nucleic Acid. Biosens. Bioelectron. 2013, 40, 258-264.

31 Zhang, X.; Li, D.; Bourgeois, L.; Wang, H.; Webley, P. A. Direct Electrodeposition of Porous Gold Nanowire Arrays for Biosensing Applications. ChemPhysChem 2009, 10, 436441.

32 Harrington, D. A.; Van den Driessche, P. Mechanism and Equivalent Circuits in Electrochemical Impedance Spectroscopy. Electrochim. Acta 2011, 56, 8005-8013. 
33 Brug, G. J.; van den Eeden, A. L. G.; Sluyters-Rehbach, M.; Sluyters, J. H. The Analysis of Electrode Impedances Complicated by the Presence of a Constant Phase Element. J. Electroanal. Chem. Interfacial Electrochem. 1984, 176, 275-295.

34 Mutha, H. K.; Lu, Y.; Stein, I. Y.; Cho, H. J.; Suss, M. E.; Laoui, T.; Thompson, C. V; Wardle, B. L.; Wang, E. N. Porosimetry and Packing Morphology of Vertically Aligned Carbon Nanotube Arrays via Impedance Spectroscopy. Nanotechnology 2017, 28, 05LT01.

35 Jurczakowski, R.; Hitz, C.; Lasia, A. Impedance of Porous Au Based Electrodes. J. Electroanal. Chem. 2004, 572, 355-366.

36 Jurczakowski, R.; Hitz, C.; Lasia, A. Impedance of Porous Gold Electrodes in the Presence of Electroactive Species. J. Electroanal. Chem. 2005, 582, 85-96.

37 Keiser, H.; Beccu, K. D.; Gutjahr, M. A. Abschätzung Der Porenstruktur Poröser Elektroden Aus Impedanzmessungen. Electrochim. Acta 1976, 21, 539-543. 


\title{
Vertically Aligned Ni Nanowires as Platform for Kinetically-Limited
}

Water Splitting Electrocatalysis

\section{SUPPORTING INFORMATION}

Minghui H. Hao ${ }^{1}$, Sébastien Garbarino ${ }^{1}$, Sagar Prabhudev², Tory Borsboom-Hanson³, Gianluigi A. Botton², David A. Harrington³ and Daniel Guay ${ }^{1 *}$

\author{
${ }^{1}$ Institut national de la recherche scientifique \\ Énergie, matériaux et télécommunications (INRS - EMT) \\ 1650 Lionel Boulet Blvd. \\ Varennes, QC, Canada J3X 1 S2 \\ 2 Department of Materials Science and Engineering and Canadian Centre for Electron Microscopy, \\ McMaster University, 1280 Main Street West, \\ Hamilton, Ontario, Canada L8S 4M4 \\ ${ }^{3}$ Department of Chemistry, \\ University of Victoria, PO Box 1700, \\ Victoria, BC, Canada V8W 2Y2
}



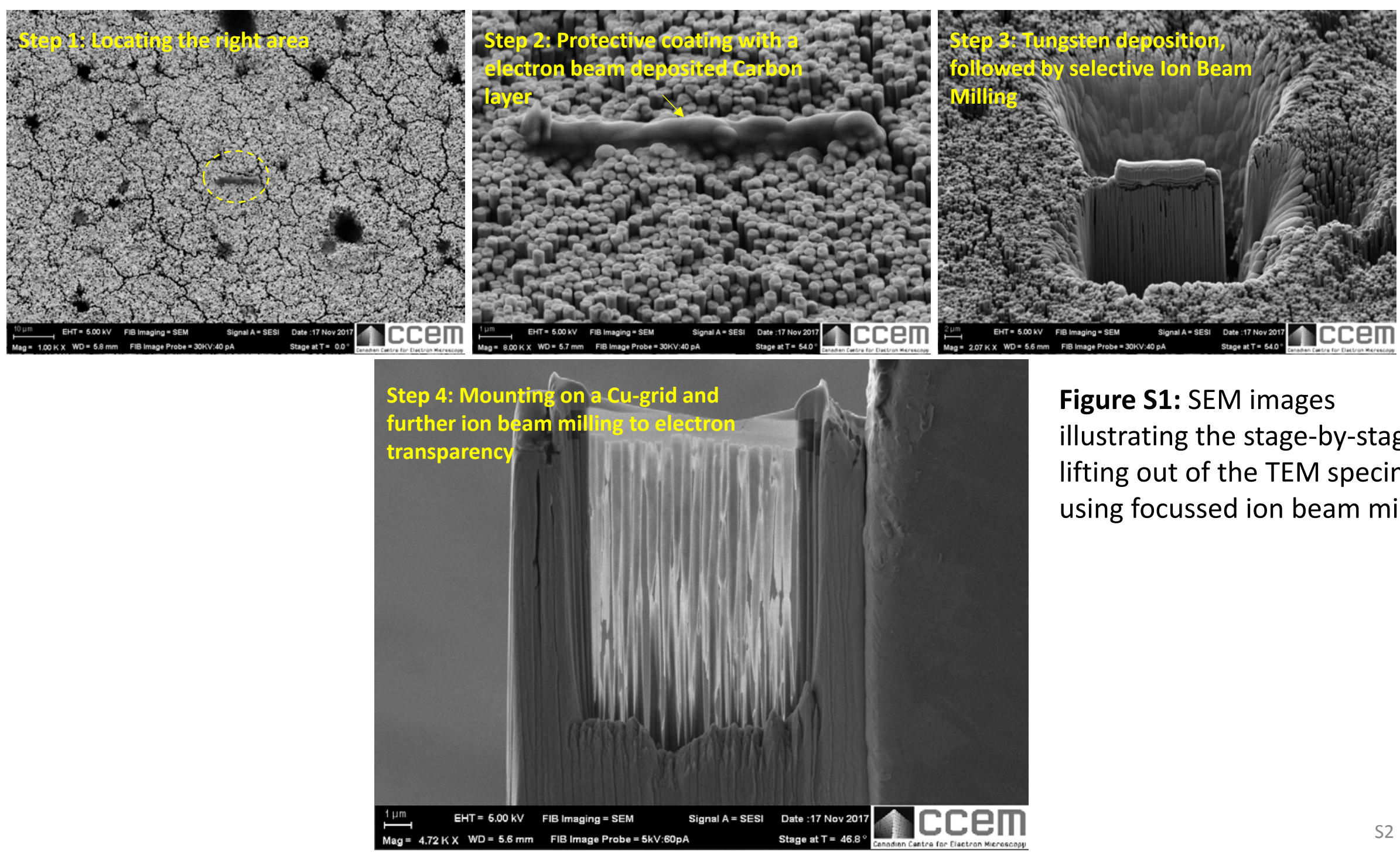

Figure S1: SEM images

illustrating the stage-by-stage lifting out of the TEM specimen using focussed ion beam milling. 
(a)
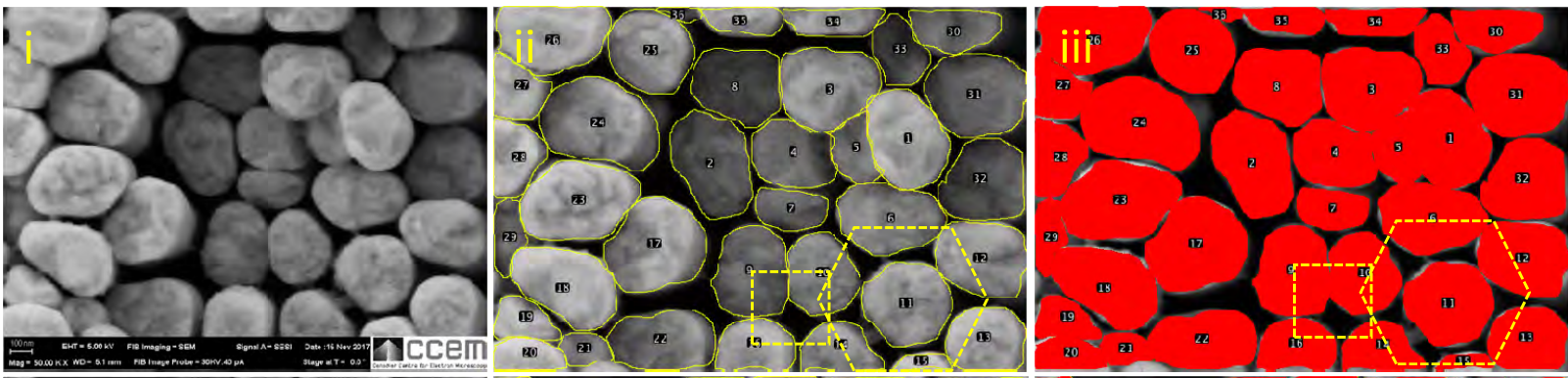

(b)
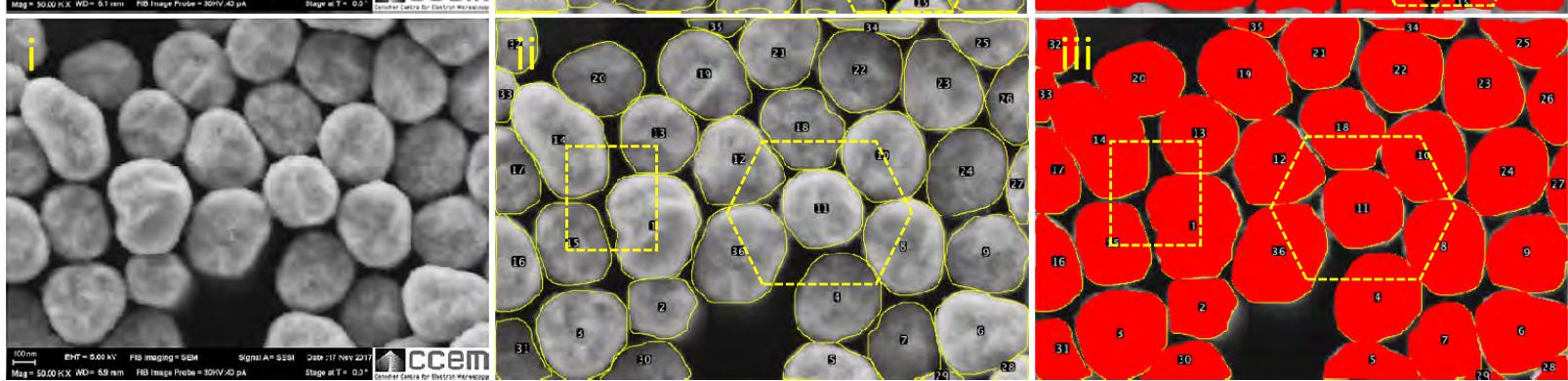

Figure S2: Estimation of packing fraction in Ni-NWs with segmentation analyses of the SEM images. (a) Pristine Ni-NWs. (b) Electrochemically Treated Ni-NWs. i, SEM Image. ii and iii, Segmented Image identifying individual NWs. (c), Comparison of the estimated packing fractions of Pristine/Treated-Ni NWs with that for the case of hexagonal and square packing of circles calculated within the error limit $<5 \%$.

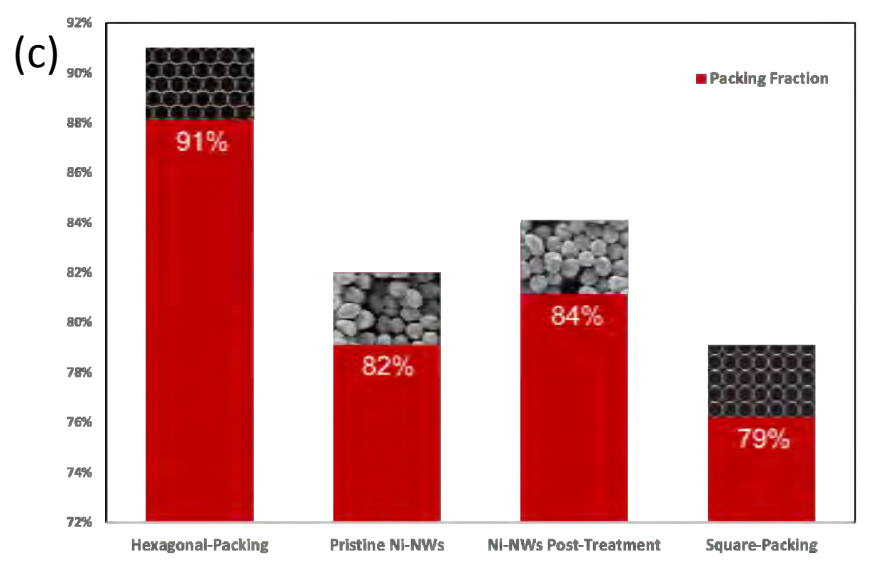




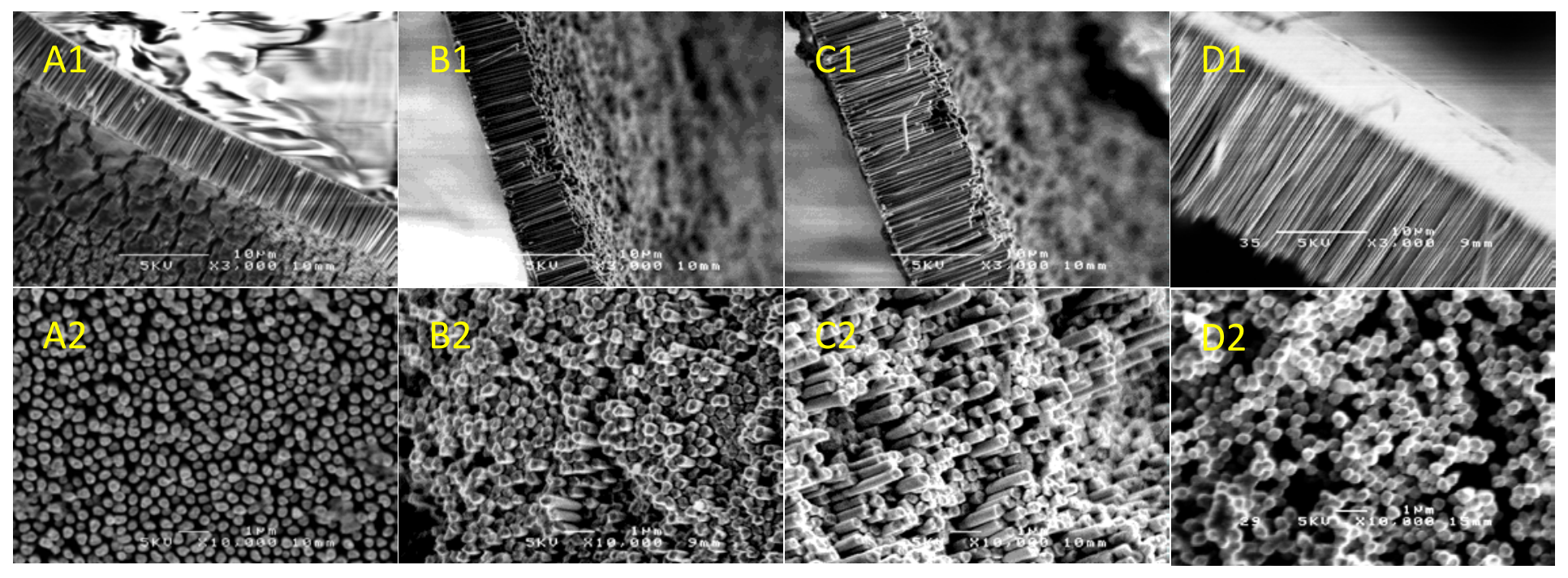

Figure S3: $\mathrm{SEM}$ micrographs for $\mathrm{Ni}$ NW arrays (A, B, C, D) with different NW lengths after electrochemical characterization in $1 \mathrm{M} \mathrm{KOH}$. A1, B1, C1, D1 - side views of NWs; A2, B2, C2, D2 - top views of NWs. The mean lengths of $A, B, C, D$ samples are $5.4 \mu \mathrm{m}, 6.6 \mu \mathrm{m}, 10.4 \mu \mathrm{m}, 17 \mu \mathrm{m}$ respectively. E, Variation of NW length and deposition current

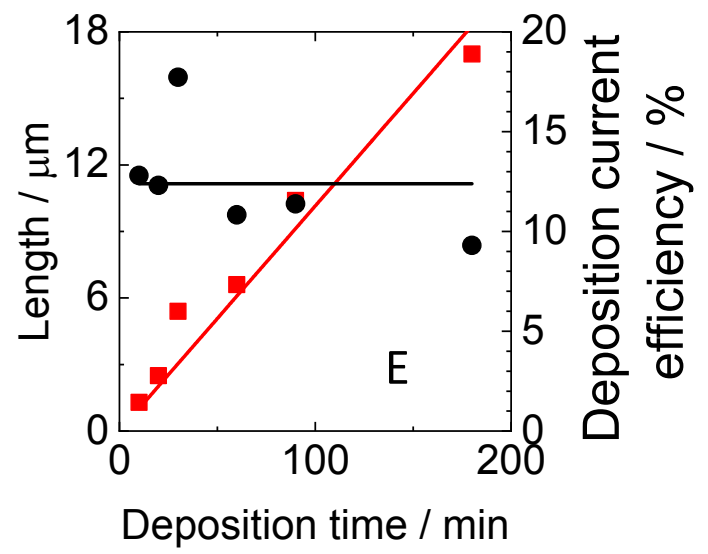
efficiency as a function of deposition time. 

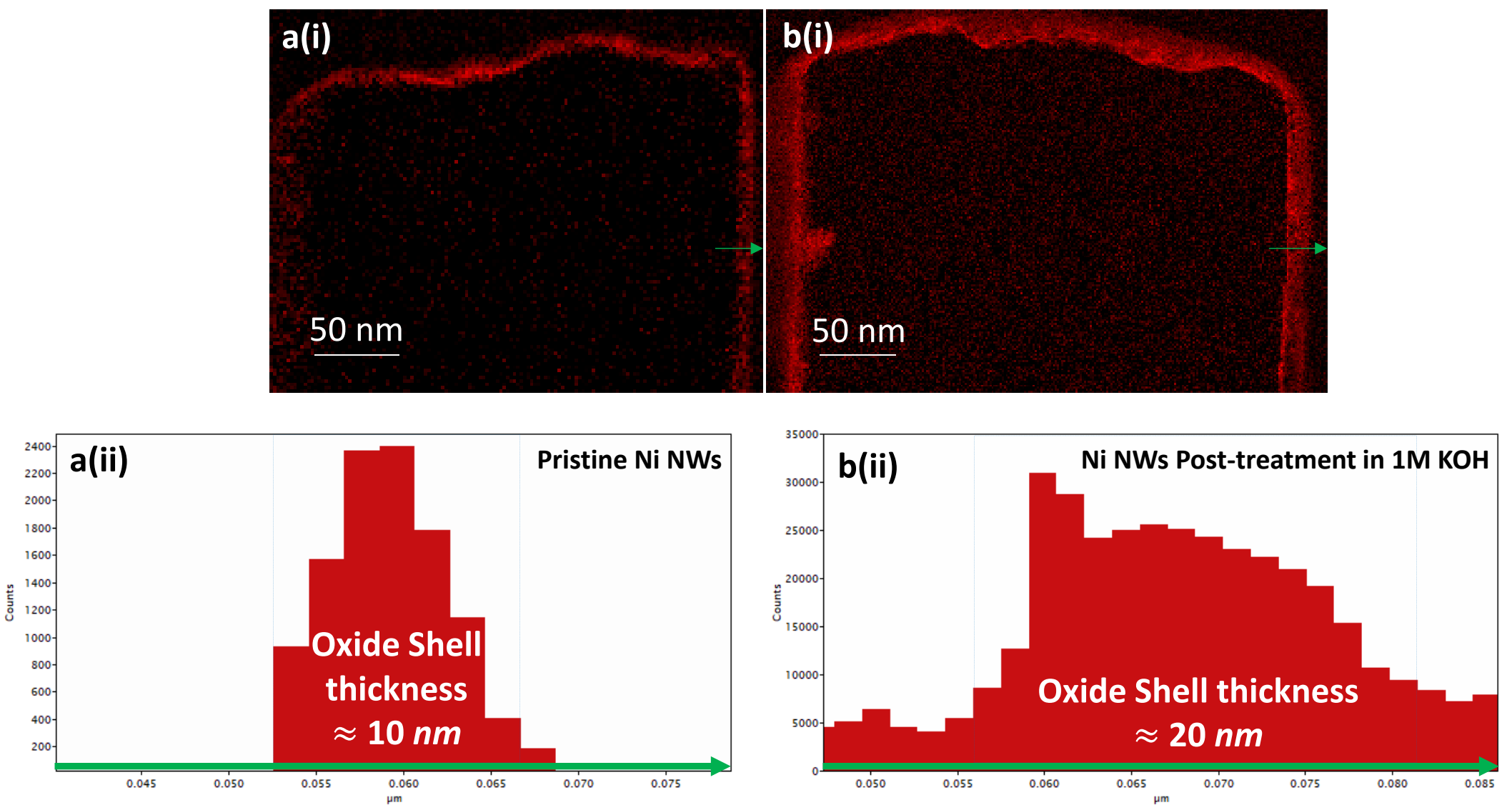

Figure S4: Spatially resolved Maps (i) and Line Profiles (ii) of the O-K edge in the case of (a) Pristine Ni NWs and (b) Ni NWs Post-treatment in $1 \mathrm{M} \mathrm{KOH}$. 


\begin{tabular}{|c|c|c|c|c|c|c|c|c|c|}
\hline Sample & E vs RHE / V & $\mathrm{Rel} / \mathrm{ohm} \mathrm{cm} \mathrm{cm}^{2}$ & $\mathrm{R} 1 / \mathrm{ohm} \mathrm{cm}^{2}$ & $\begin{array}{c}\mathrm{T} 1 / \Omega^{-1} \mathrm{~cm}^{-2} \varsigma^{\varnothing} \\
\times 10^{-3}\end{array}$ & $\varnothing 1$ & Rct / ohm cm² & $\begin{array}{c}\text { Teff / } \Omega^{-1} \mathrm{~cm}^{-2} \\
S^{\varnothing} \times 10^{-3}\end{array}$ & $\varnothing$ eff & 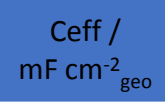 \\
\hline \multirow{6}{*}{ Ni plate } & 1.49 & $4.76 \pm 0.05$ & & & & $1979 \pm 94$ & $1.47 \pm 0.02$ & $0.873 \pm 0.003$ & $0.72 \pm 0.02$ \\
\hline & 1.51 & $4.74 \pm 0.05$ & & & & $736 \pm 17$ & $1.38 \pm 0.02$ & $0.871 \pm 0.004$ & $0.65 \pm 0.02$ \\
\hline & 1.53 & $4.73 \pm 0.05$ & & & & $307 \pm 4$ & $1.35 \pm 0.02$ & $0.863 \pm 0.004$ & $0.60 \pm 0.02$ \\
\hline & 1.55 & $4.69 \pm 0.03$ & & & & $148 \pm 1$ & $1.32 \pm 0.02$ & $0.856 \pm 0.004$ & $0.56 \pm 0.02$ \\
\hline & 1.57 & $4.66 \pm 0.03$ & & & & $84.2 \pm 0.5$ & $1.31 \pm 0.02$ & $0.848 \pm 0.003$ & $0.52 \pm 0.01$ \\
\hline & 1.59 & $4.62 \pm 0.03$ & & & & $56.1 \pm 0.3$ & $1.31 \pm 0.02$ & $0.835 \pm 0.004$ & $0.47 \pm 0.02$ \\
\hline \multirow{6}{*}{ NW $2.5 \mu \mathrm{m}$} & 1.49 & $7.28 \pm 0.03$ & $4.47 \pm 0.11$ & $4.63 \pm 0.33$ & $0.71 \pm 0.01$ & $931 \pm 36$ & $9.89 \pm 0.03$ & $0.946 \pm 0.003$ & $8.50 \pm 0.08$ \\
\hline & 1.51 & $7.29 \pm 0.03$ & $4.44 \pm 0.09$ & $3.78 \pm 0.27$ & $0.73 \pm 0.01$ & $443 \pm 7$ & $9.01 \pm 0.03$ & $0.947 \pm 0.002$ & $7.74 \pm 0.05$ \\
\hline & 1.53 & $7.30 \pm 0.03$ & $4.46 \pm 0.09$ & $3.53 \pm 0.27$ & $0.73 \pm 0.01$ & $204 \pm 2$ & $8.53 \pm 0.03$ & $0.949 \pm 0.002$ & $7.33 \pm 0.05$ \\
\hline & 1.55 & $7.30 \pm 0.03$ & $4.56 \pm 0.11$ & $3.71 \pm 0.27$ & $0.72 \pm 0.01$ & $105.4 \pm 0.6$ & $8.19 \pm 0.03$ & $0.949 \pm 0.003$ & $7.02 \pm 0.07$ \\
\hline & 1.57 & $7.29 \pm 0.03$ & $4.55 \pm 0.12$ & $3.93 \pm 0.33$ & $0.71 \pm 0.01$ & $61.7 \pm 0.4$ & $8.11 \pm 0.04$ & $0.946 \pm 0.004$ & $6.85 \pm 0.10$ \\
\hline & 1.59 & $7.18 \pm 0.03$ & $4.64 \pm 0.15$ & $5.94 \pm 0.47$ & $0.64 \pm 0.01$ & $40.2 \pm 0.2$ & $7.99 \pm 0.05$ & $0.941 \pm 0.004$ & $6.62 \pm 0.10$ \\
\hline \multirow{6}{*}{ NW $17 \mu \mathrm{m}$} & 1.49 & $9.07 \pm 0.02$ & $2.90 \pm 0.03$ & $2.96 \pm 0.20$ & $0.86 \pm 0.01$ & $316 \pm 30$ & $60.09 \pm 0.13$ & $0.951 \pm 0.004$ & $58.16 \pm 0.21$ \\
\hline & 1.51 & $8.98 \pm 0.02$ & $2.82 \pm 0.03$ & $2.63 \pm 0.20$ & $0.88 \pm 0.01$ & $180 \pm 8$ & $54.37 \pm 0.13$ & $0.952 \pm 0.004$ & $52.30 \pm 0.22$ \\
\hline & 1.53 & $9.00 \pm 0.02$ & $2.77 \pm 0.03$ & $2.68 \pm 0.20$ & $0.87 \pm 0.01$ & $96 \pm 2$ & $50.45 \pm 0.13$ & $0.945 \pm 0.004$ & $47.93 \pm 0.23$ \\
\hline & 1.55 & $9.02 \pm 0.02$ & $2.73 \pm 0.05$ & $2.68 \pm 0.20$ & $0.87 \pm 0.01$ & $58 \pm 1$ & $45.59 \pm 0.13$ & $0.921 \pm 0.004$ & $41.71 \pm 0.24$ \\
\hline & 1.57 & $9.02 \pm 0.02$ & $2.80 \pm 0.03$ & $2.73 \pm 0.13$ & $0.87 \pm 0.01$ & $35.8 \pm 0.3$ & $44.90 \pm 0.13$ & $0.942 \pm 0.003$ & $41.86 \pm 0.21$ \\
\hline & 1.59 & $9.02 \pm 0.02$ & $2.70 \pm 0.05$ & $2.78 \pm 0.20$ & $0.86 \pm 0.01$ & $24.6 \pm 0.2$ & $46.03 \pm 0.20$ & $0.948 \pm 0.004$ & $43.14 \pm 0.30$ \\
\hline
\end{tabular}

Table S1 : Fitting parameters of different electrodes obtained by using the corresponding equivalent circuits shown in Figure 10. 


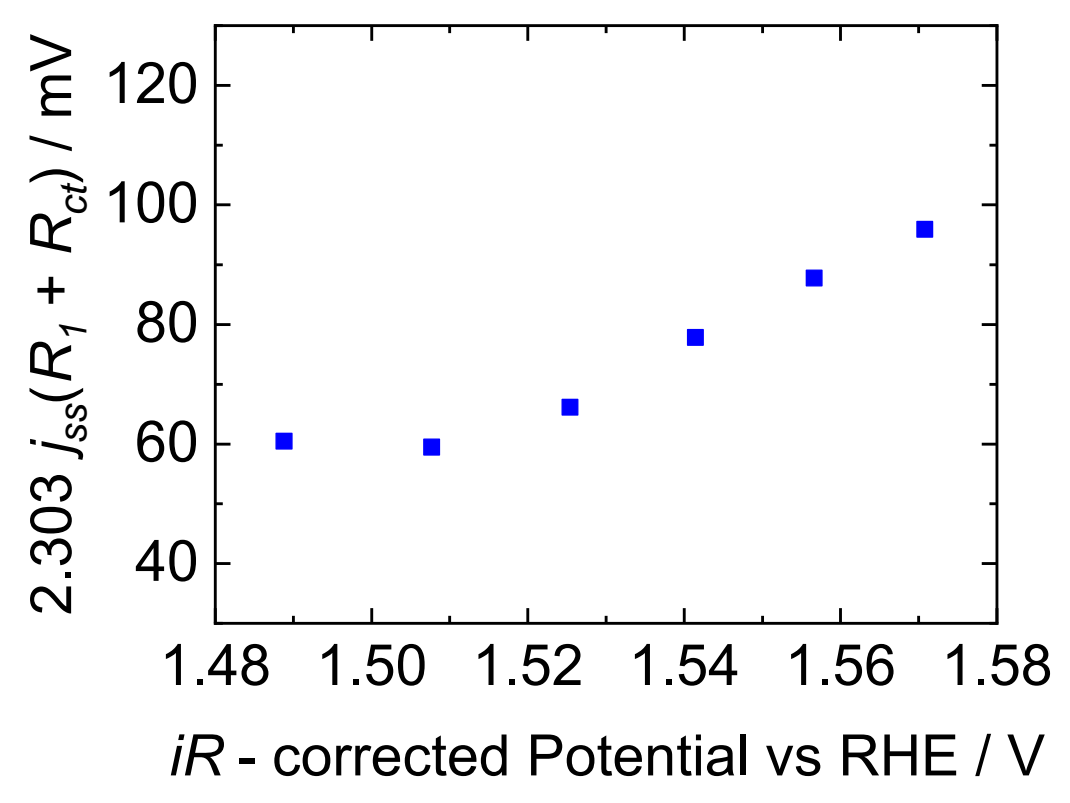

Figure S5 : Product of Steady State Current Density and $R_{p}=R_{1}+R_{c t}$ as a function of potentials on $17 \mu \mathrm{m}$ Ni NWs. 


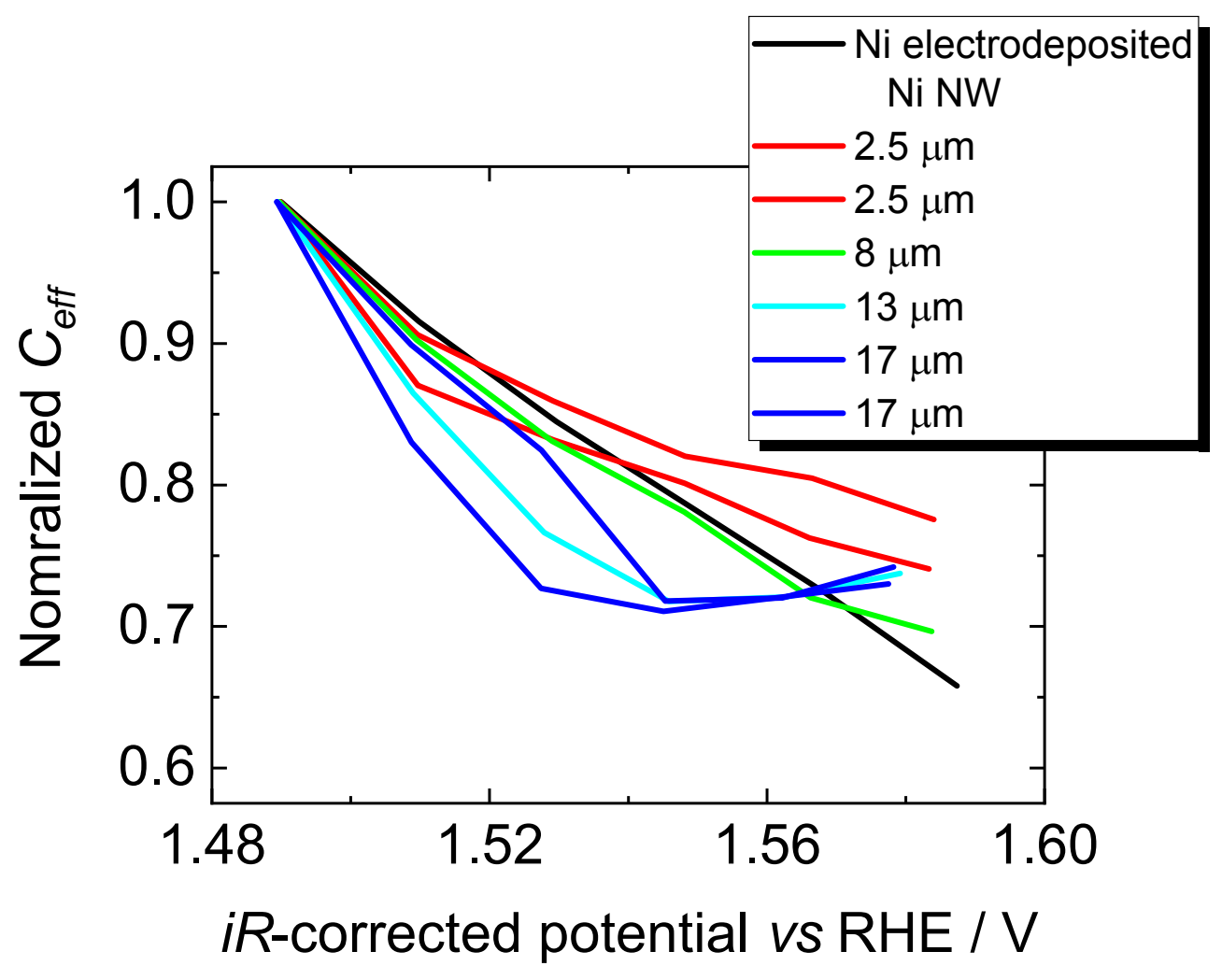

Figure S6 : Normalized effective capacitance in function of potential. The $C_{\text {eff }}$ value at $1.49 \mathrm{~V}$ of each electrode is used as reference. 


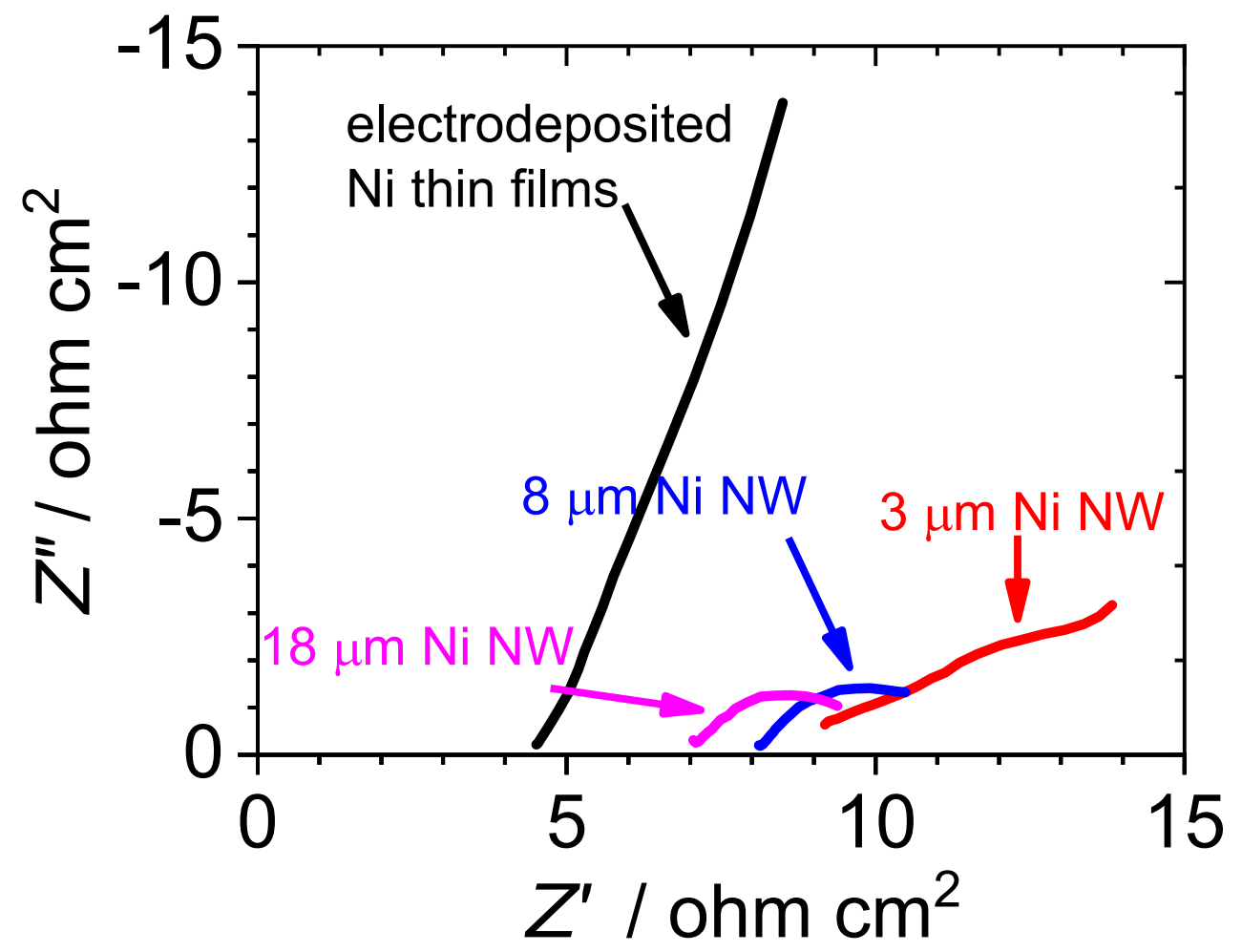

Figure S7 : High-frequency part of the IES spectra recorded at $1.49 \mathrm{~V}$ vs RHE. 